\title{
OPEN Mesenchymal stromal cell mitochondrial transfer to human induced T-regulatory cells mediates FOXP3 stability
}

\author{
Jeong-su Do ${ }^{1 \bowtie}$, Daniel Zwick², Jonathan D. Kenyon², Fei Zhong ${ }^{2}$, David Askew ${ }^{3}$, \\ AlexY. Huang ${ }^{3}$, Wouter Van't Hof ${ }^{2}$, Marcie Finney ${ }^{2}$ \& Mary J. Laughlin ${ }^{2,4}$
}

The key obstacle to clinical application of human inducible regulatory T cells (iTreg) as an adoptive cell therapy in autoimmune disorders is loss of FOXP3 expression in an inflammatory milieu. Here we report human iTreg co-cultured with bone marrow-derived mesenchymal stromal cells (MSCs) during short-term ex vivo expansion enhances the stability of iTreg FOXP3 expression and suppressive function in vitro and in vivo, and further that a key mechanism of action is MSC mitochondrial (mt) transfer via tunneling nanotubules (TNT). MSC mt transfer is driven by mitochondrial metabolic function (CD39/CD73 signaling) in proliferating iTreg and promotes iTreg expression of FOXP3 stabilizing factors $\mathrm{BACH} 2$ and SENP3. These results elucidate cellular and molecular mechanisms underlying human MSC mt transfer to proliferating cells. MSC mt transfer stabilizes FOXP3 expression in iTregs, thereby enhancing and sustaining their suppressive function in inflammatory conditions in vitro and in vivo.

Human and murine CD4 T cells can be efficiently differentiated ex vivo in the presence of TGF- $\beta$ to generate inducible regulatory $\mathrm{T}$ cells (iTregs) with equivalent suppressive capacity as thymus-derived T regulatory cells $(\mathrm{t} \text { Treg })^{1,2}$. iTregs have potentially enormous clinical application in diverse inflammatory conditions, including autoimmune disorders and graft versus host disease ${ }^{3}$. However, the instability of FOXP3 expression in iTregs, resulting in loss of suppressive function in inflammatory conditions, has to date limited wide clinical application ${ }^{4}$.

A number of studies have shown tTreg cells prevent adverse inflammation in multiple disease settings including autoimmunity and graft vs. host disease $\mathrm{e}^{5,6}$. Although ex vivo protocols to expand tTregs have been developed, the limited number and anergic nature of tTregs, the risk of effector T cell contamination during expansion ${ }^{7-9}$, and the instability of tTreg phenotype and suppressor function upon transfer to an inflammatory environment ${ }^{10,11}$, remain significant obstacles to clinical efficacy.

Treg suppressive function is tightly controlled by Foxp3 co-transcriptional proteins ${ }^{12,13}$. BACH2, a Broadcomplex-tramtrack-Bric-a-Brac and Cap'n'collar homology 2 bZip transcription factor, has been shown to stabilize Treg Foxp3 expression in recent murine studies ${ }^{14,15}$. BACH2 has also been shown to regulate FOXP3 expression in human Umbilical Cord Blood (UCB) iTreg ${ }^{16}$. Further, murine studies identify SUMO specific protease 3 (SENP3) to be a critical regulator of Treg function by regulating the SUMOylation and nuclear localization of $\mathrm{BACH} 2^{17}$.

Expansion of T cells in IL-2 based standard suspension cultures in vitro is associated with cell differentiation and senescence ${ }^{18}$. We observed previously, the addition of feeder layer MSCs provides improved cytokine driven expansion of early UCB CD34(+)/CD38(-)/HLA-DR(-) hematopoietic progenitors and inhibits differentiation and rates of apoptosis during short-term cytokine driven in vitro expansion ${ }^{19}$. Further, previous work has shown that MSC improve recovery of injured neuronal cells via mitochondrial transfer ${ }^{20,21}$. MSC have been identified to favor iTreg formation ${ }^{22}$, yet the potential of MSC to promote iTreg functional stabilization has not been explored.

\footnotetext{
${ }^{1}$ Department of Immunology and Theranostics, Diabetes and Metabolism Research Institute, The Beckman Research Institute, City of Hope National Medical Center, 1500 E. Duarte Road, Duarte, CA 91010, USA. ${ }^{2}$ Cleveland Cord Blood Center, 25001 Emery Rd, Cleveland, OH 44106, USA. ${ }^{3}$ Department of Pediatrics, School of Medicine, Case Western Reserve University, Cleveland, $\mathrm{OH} 44106$, USA. ${ }^{4}$ Department of Biomedical Engineering, School of Medicine, Case Western Reserve University, Health Education Campus, 9501 Euclid Ave, Cleveland, OH 44106, USA. ${ }^{\boxplus}$ email: jedo@coh.org
} 
Our intent with this study was to improve upon the current standard in the field of Treg expansion for clinical use, e.g. short-term 3 week IL-2 based suspension culture conditions. We examined whether iTreg co-culture over a mono-layer of mesenchymal stromal cells (MSCs) could minimize iTreg cell differentiation and senescence and enhance iTreg number and suppressive function during in vitro expansion vs. standard IL-2 media suspension culture conditions ${ }^{23,24}$.

\section{Results}

MSC co-culture renders iTreg stability. Human marrow MSC exhibited morphology and phenotype (CD44, CD73, CD90 and CD105 expression) as previously described ${ }^{25}$ (Supplementary Fig. S1a). UCB-derived naive $C D 4^{+} C D 45 R A^{+} T$ cells were differentiated into iTreg in standard 4 day culture in TGF- $\beta$ condition ${ }^{16}$. UCB FOXP3 ${ }^{+}$iTregs co-cultured with MSCs rendered a significantly higher percentage and absolute number of FOXP $^{+}$iTregs compared to standard IL-2/media suspension culture condition ${ }^{26}$ at day 14 and 21 and show similar viability (Fig. $1 \mathrm{~b}$ and Supplementary Fig. 1b-d). We also examined CD62L and CD45RA, markers associated with Treg subsets with enhanced suppressive capacity ${ }^{27,28}$ and naive phenotype ${ }^{29}$. CD62L $\mathrm{L}^{+}$and CD45RA ${ }^{+}$iTregs were significantly increased during 21 day culture over MSCs (Fig. 1c and Supplementary Fig. 1e). Although prior studies point to paracrine secretion of factors by MSC as sufficient to enhance iTreg induction ${ }^{30}$, we did not observe enhanced FOXP3 expression via MSC-mediated paracrine effect in a trans-well system (Fig. $1 \mathrm{~b}$ and Supplementary Fig. 1d). Intriguingly, iTregs co-cultured with MSC exhibited significantly higher FOXP3 expression (Supplementary Fig. 1d). We next investigated the impact of MSC feeder layer co-culture on expression of surface molecules involved in iTreg inhibitory function and any evidence of cell exhaustion. As shown in Fig. 1c, MSC co-culture was associated with significantly enhanced expression of several molecules on iTregs associated with suppressive $\mathrm{T}$ cell function, e.g. CD25, CD152, and CD278 ${ }^{31}$. MSC platform iTregs also had significantly lower expression of T cell exhaustion surface markers (LAG-3, TIM-3) during short-term (21 day) culture in IL-2 (Fig. 1d). These observations suggest that MSC modulate iTreg phenotype and function during IL-2 mediated short-term expansion. Of note, expression of these iTreg markers was not observed in naive CD4 T cells on Day 0 prior to 4 day Treg induction (Fig. 1d). Robust expression of these markers was observed by Day 4 of iTreg differentiation, immediately prior to splitting into short-term expansion in IL-2/media suspension culture vs. identical IL-2/media on a monolayer of MSC feeder cells (Supplementary Fig. 1f,g). In further support of a role for MSC in Treg stabilization, we observed that MSC also enhance maintenance of Treg marker expression in tTregs isolated from UCB during 21 days of culture expansion over MSC (Supplementary Fig. 1g,h).

Next, we tested whether MSC co-culture enhances iTreg suppressive function ${ }^{32}$. MSC co-culture dramatically enhanced CD $25^{+}$isolated iTreg suppression of CD4 T cell proliferation in vitro (Fig. $2 \mathrm{a}$ and Supplementary Fig. 2a,b). A pronounced enhancement of iTreg suppression was observed even at very low 10:1 and 50:1 ratios compared with iTreg expanded in IL-2/media suspension culture conditions.

Inflammatory conditions destabilize iTreg phenotype and downregulate suppressive function ${ }^{33-35}$. We restimulated iTregs in the presence of pro-inflammatory cytokines to mimic an inflammatory environment and measured FOXP3 stability. MSC co-culture stabilized iTregs in this in vitro inflammatory environment (Fig. 2b). FOXP3 expression in MSC co-culture expanded iTregs remained $>90 \%$ when in resting state $(n=3$, data not shown) in further support of enhanced iTreg stability. These data suggest that MSC feeder cells preserve iTreg FOXP3 expression and suppressive function during IL-2 driven short-term (21 day) expansion.

MSC mt transfer requires direct cell-cell contact. Intercellular mt transfer by MSC has been previously described in neuronal injury and cancer models $\mathrm{s}^{36}$. We measured total $\mathrm{mt}$ content in iTreg cells following MSC co-culture by flow cytometric analysis of iTregs labeled with the cardiolipin-binding dye nonyl acridine orange $(\mathrm{NAO})^{37}$. MSC co-culture induced a $\sim$ twofold increase in NAO mean fluorescence intensity in iTregs (Fig. 3a). To determine whether this resulted from increased endogenous iTreg mt biogenesis or MSC mt transfer, we labeled MSC mt with MitoTracker and enumerated MitoTracker positive iTreg in MSC co-cultures by direct visualization using epifluorescence microscopy. Intriguingly, robust mt transfer was observed (Fig. 3b), as the proportion of MitoTracker ${ }^{+}$iTregs reached $\sim 16 \%$ within $16 \mathrm{~h} \mathrm{MSC} \mathrm{co-culture} \mathrm{(Supplementary} \mathrm{Fig.} \mathrm{3a).}$ Expanded iTreg mt copy number was further increased in iTregs during short-term (21 day) expansion in MSC co-culture condition (Fig. 3c). Mitochondrial transfer was directly visualized via tunneling nanotubules (TNT) between MSCs and iTregs (Fig. 3d and Supplementary Fig. 3b,c). To determine whether MSC mt transfer was reliant on TNT for transfer to proliferating iTreg during IL-2 driven expansion culture, Cytochalasin B (Cyto B), an inhibitor of TNT formation, was added and completely blocked the transfer of MitoTracker ${ }^{+} \mathrm{mt}_{\text {from MSC }}$ to proliferating Tregs (Fig. 4a,b and Supplementary Fig. 3d). Of note, Cytochalasin B was utilized at a concentration $(350 \mathrm{nM})$ known to inhibit TNT formation with minimal effects on cell viability in a range of cells including MSC, acute myeloid leukemia cells, and PC12 cells $^{38,39}$. Nevertheless, one possibility is that reduced mt transfer was mediated by cyto B induced cytotoxicity in MSCs. Thus, we measured apoptosis by Annexin V (AV) staining. No differences in the proportion of $\mathrm{AV}^{+} \mathrm{MSCs}$ (Fig. 4c), or in $\mathrm{AV}+$ Tregs (Supplementary Fig. 3e) between mock and cyto B treatment conditions was observed. Further, pre-treatment of MSCs, but not iTregs, with cyto $\mathrm{B}$ inhibited $\mathrm{mt}$ transfer, indicating MSC mt transfer occurs via TNT formation from MSCs (Supplementary Fig. 3f,g). We next tested the impact of MSC mt transfer on FOXP3 stability in iTregs. Cytochalasin B treatment significantly reduced FOXP3 expression in iTregs expanded on MSC (Fig. 4d).

Cellular reactive oxygen species (ROS) are implicated as regulators of MSC mt transfer in cancer ${ }^{39}$. We incorporated treatment with the ROS scavenger NAC ( $N$-acetyl-L-cysteine) and observed an incomplete block of MSC mt transfer (Fig. 4e). As mt transfer can be induced by MSC derived soluble and exosome-mediated

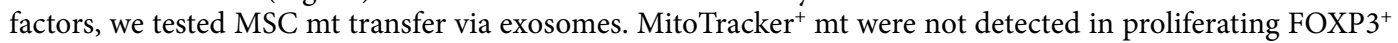
iTregs in IL2/media in the presence of MSC exosomes (Fig. 4f). Further, using a transwell separating MSCs from 
a

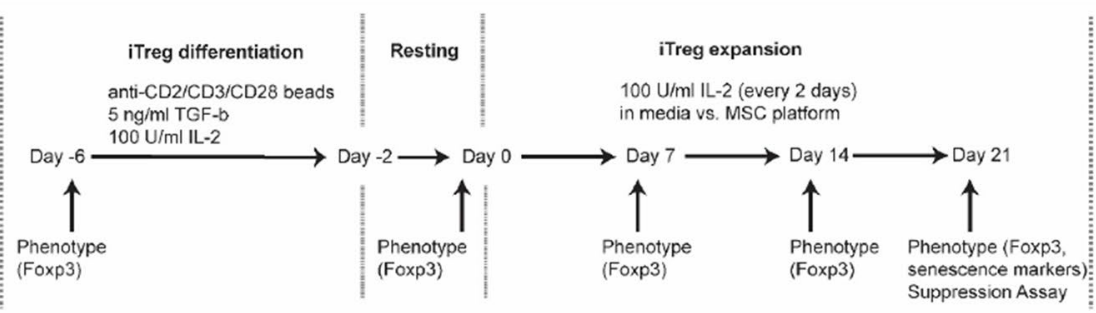

b

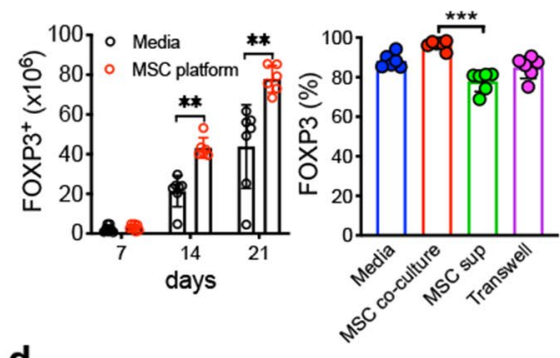

d

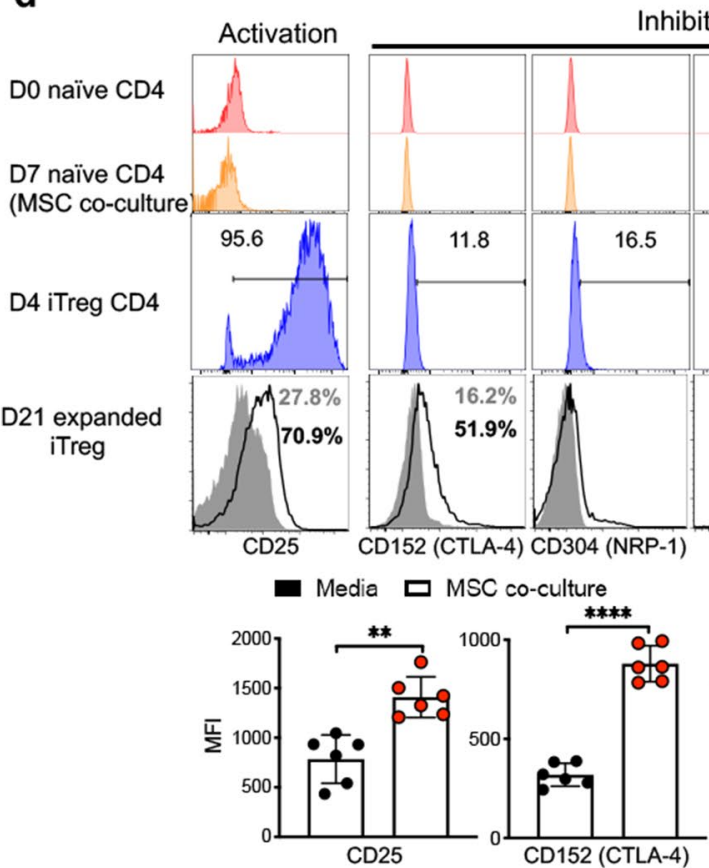

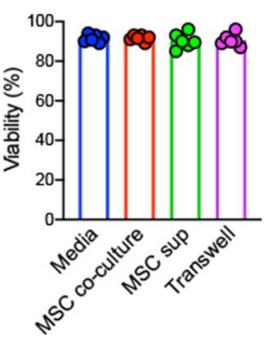

nhibitory

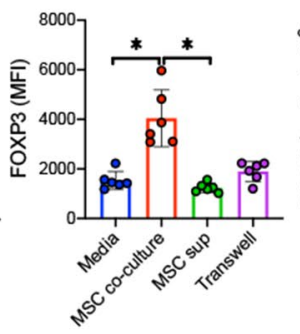

C

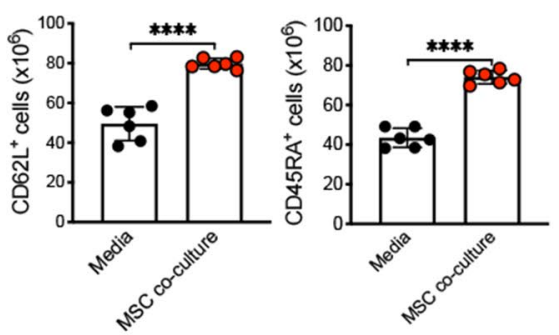

Exhaustion

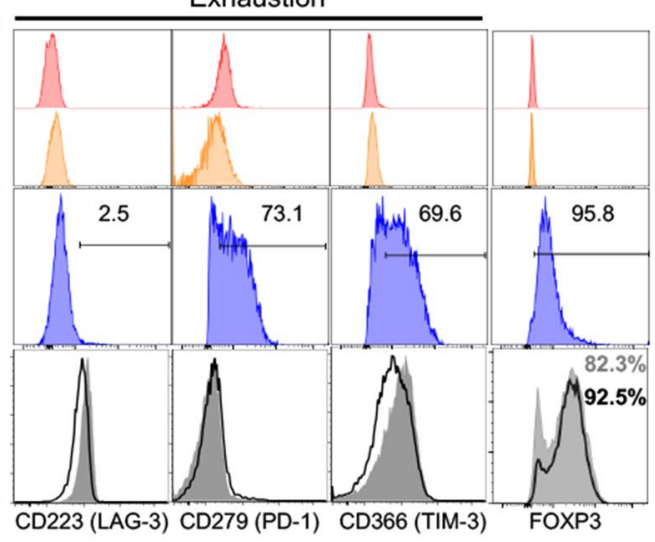

Figure 1. MSCs enhance and sustain intrinsic iTreg phenotype during IL-2 driven ex vivo expansion. (a) Schematic diagram shows experimental strategy for iTreg expansion. (b) Absolute number, percentage of FOXP3 $^{+}$, FOXP3 MFI in CD4 T cells and viability of CD4 T cells at day 21 were measured at indicated time points in IL-2/media vs MSC co-culture with identical IL-2/media $(n=6-7)$. Multiple comparisons analysis was performed using the Friedman test. (c) Absolute number of CD62 $\mathrm{L}^{+}$and CD45RA ${ }^{+}$iTreg cells were calculated at 21 days expansion $(n=5-6)$. (d) Immuno-phenotyping of Treg marker expression on D0 naïve CD4 T cells, 7 days expanded naïve T cells in IL-2/media over MSC monolayer w/o CD3/CD28 stimulation (Day 7 naïve CD4-MSC co-culture), or 4 days stimulated CD 4 T cells with TGF- $\beta / \mathrm{IL}-2$ and CD3/28-(Day 4 iTreg CD4) and iTregs included surface staining with antibodies targeting CD25, CD152 (CTLA-4), CD223 (LAG-3), CD278 (ICOS) CD304 (NRP-1), CD279 (PD-1), CD366 (Tim-3), and TIGIT at 21 days expansion. Data are representative of three independent experiments \pm SD. ${ }^{*} p<0.05,{ }^{* *} p<0.01,{ }^{* * *} p<0.001,{ }^{* * * *} p<0.0001$ paired $t$ test. See also Supplementary Fig. S1.

iTregs, MitoTracker ${ }^{+}$iTregs were not observed, suggesting direct cell-cell contact is necessary for efficient $\mathrm{mt}$ transfer (Supplementary Fig. 3d). In addition, MSC mt transfer to iTregs did not occur in quiescent iTreg and is dependent on iTreg proliferation driven by IL-2 (Supplementary Fig. 4a,b). It has been shown other mesenchymal 
a

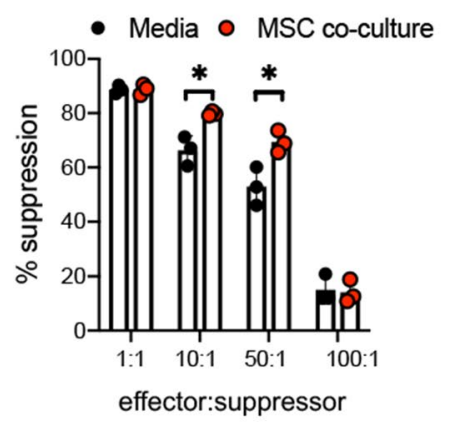

b

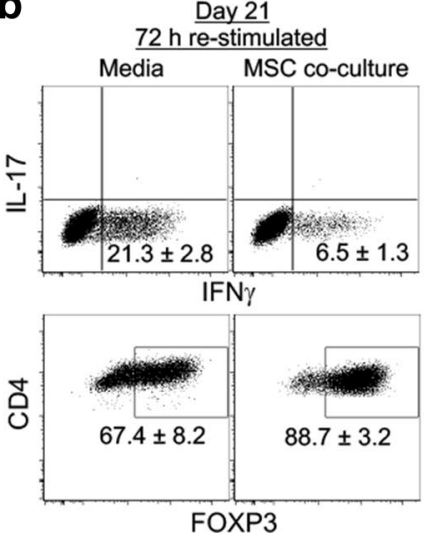

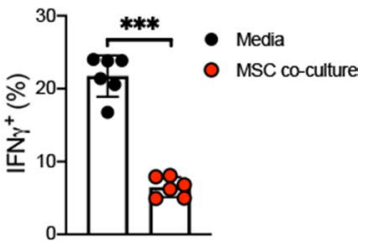

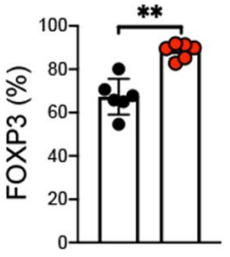

Figure 2. MSC co-culture stabilises FOXP3 expression in iTregs in the presence of inflammatory cytokines. (a) Suppressive effects of iTregs were measured day 21 of ex vivo expansion of iTregs in IL-2/media vs MSC co-culture, CD25 ${ }^{\text {high }}$ FACS sorted iTregs were used to test suppressive function. 4 days after stimulation, CFSE dilution was examined by FACS $(n=3)$. (b) Expression of FOXP3 on iTregs was measured following $72 \mathrm{~h}$ restimulation with CD3 and CD28 antibodies $\left(1 \mu \mathrm{g} \mathrm{ml} l^{-1}\right)$ in the presence of inflammatory cytokines IFN $\gamma$ $\left(10 \mu \mathrm{g} \mathrm{ml}^{-1}\right)$, IL-6 $\left(10 \mu \mathrm{g} \mathrm{ml}^{-1}\right)$, and TNF $\left(10 \mu \mathrm{g} \mathrm{ml}^{-1}\right)$ at concentration of $5 \times 10^{5} \mathrm{cells} \mathrm{ml}^{-1}$ and analyzed by FACS. Intracellular IFN $\gamma$ and IL-17 were detected by flow cytometry analysis. Data are representative of two independent experiments \pm SD $(n=6-7){ }^{\star *} p<0.01,{ }^{\star * *} p<0.001$ paired t test. See also Supplementary Fig. S2.

a
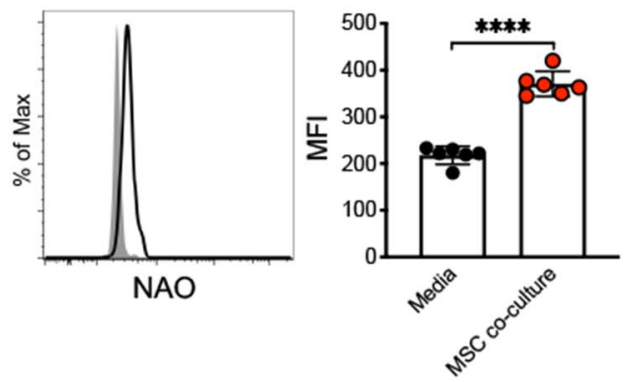

C

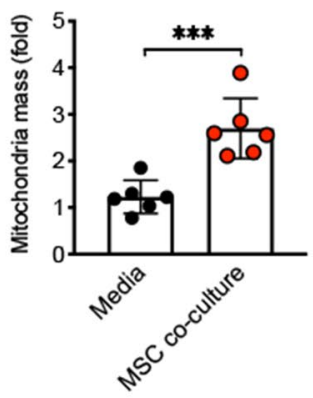

d
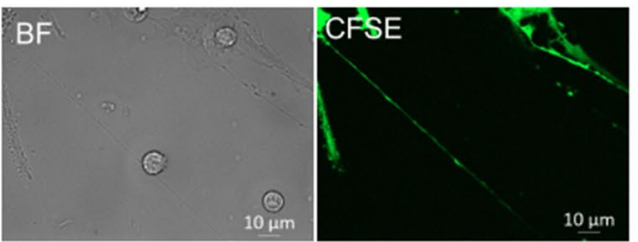

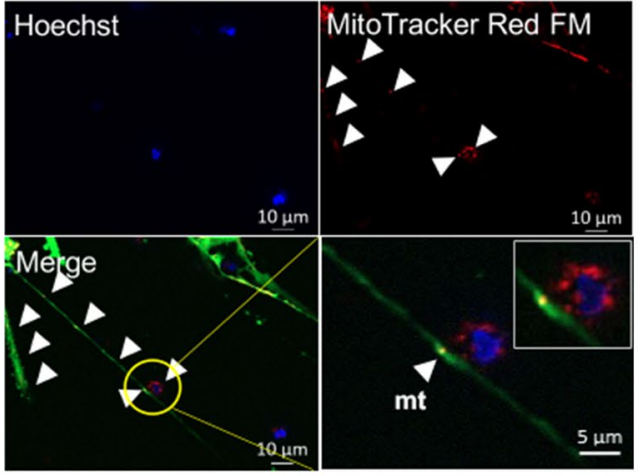

b

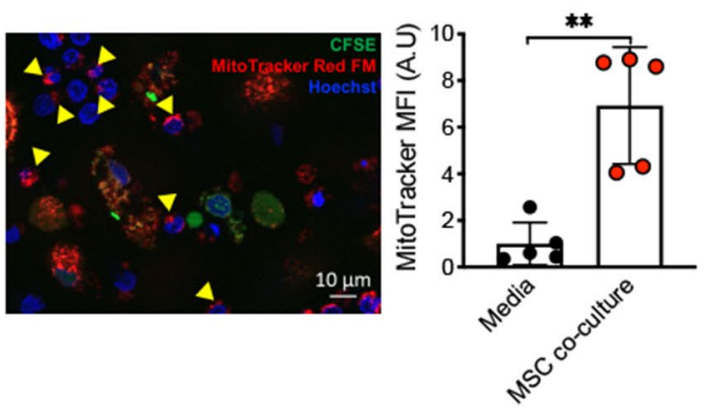

Figure 3. MSC mitochondrial transfer to iTregs occurs via TNT. (a) Mitochondrial quantity assay of iTregs was analyzed. At 3 weeks, media vs MSC co-culture iTreg cells were stained with NAO to quantify mt mass $(n=5)$. (b) Confocal imaging analysis for MitoTracker transfer. MSC were stained with CFSE (green) and MitoTracker Far Red (red), iTreg were stained with Hoechst (blue) and then co-cultured for 24-36 h $(n=5)$. (c) Mitochondria DNA copy number was assessed from IL-2/media or MSC co-culture expanded iTregs at day $21(n=6)$. (d) MSC $\mathrm{mt}$ transfer analysis. MSC were stained with CFSE (green) and MitoTracker Far Red (red), iTreg were stained Hoechst (blue) and cells were co-cultured for 24-36 h. Live cell images were collected by confocal microscopy. Data are representative of three independent experiments. (b,d) Analysis of recorded images was performed using Zen software Blue edition, (2011; version 2.0.14283.302). https://www.zeiss.com/microscopy/us/produ cts/microscope-software/zen.html ${ }^{* *} p<0.01,{ }^{* *} p<0.001,{ }^{* * * *} p<0.0001$ paired t test. Scale bars: $10 \mu \mathrm{m}$. See also Supplementary Fig. S3. 
a

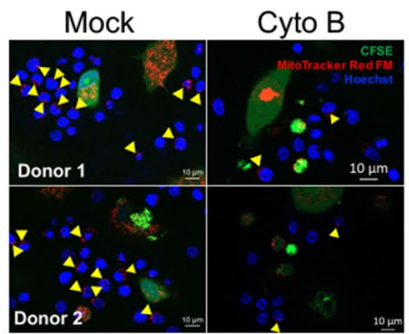

c

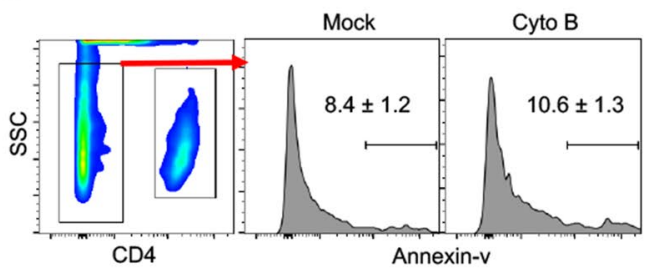

e

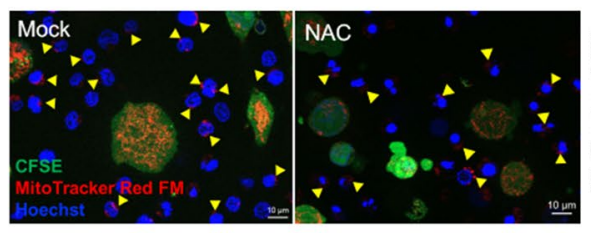

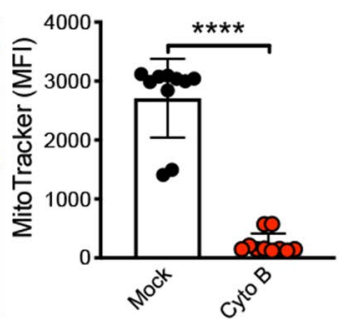

cto

b
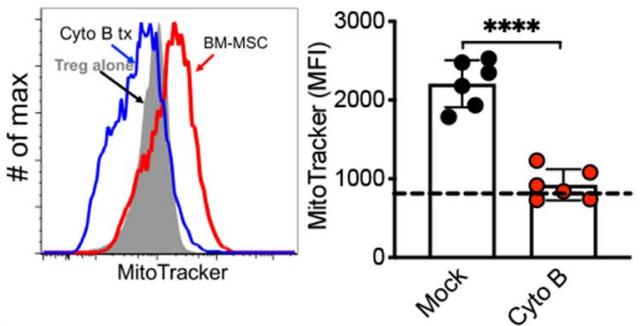

d
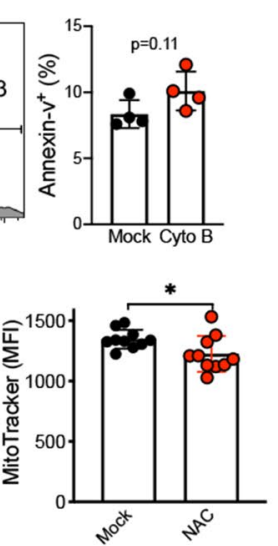

Media
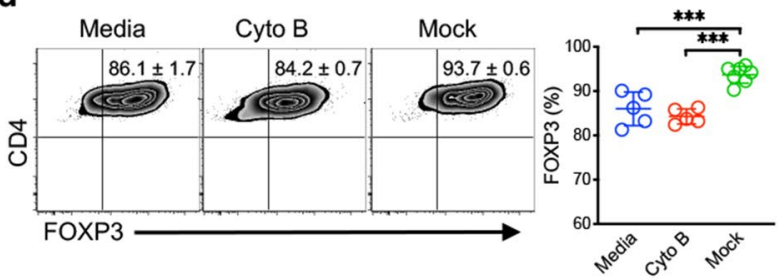

f

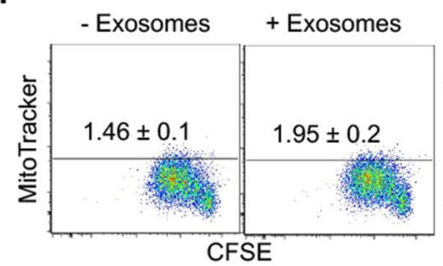

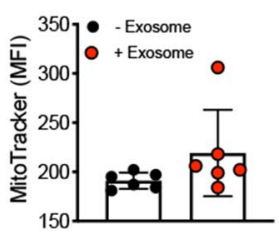

Figure 4. MSC-iTreg interactions are required for $\mathrm{mt}$ transfer. (a) Mitochondrial transfer measured in iTreg co-cultured with cytochalasin B treated MSC for 24-36 h $(n=5)$. Cytochalasin B was added in the MSC co-culture $(350 \mathrm{nM})$. Arrowheads indicate $\mathrm{mt}$ transfer to iTreg. Analysis of recorded images was performed using Zen 2012 software. (b) FACS analysis of MSC mt transfer to iTreg after cytochalasin B treatment. MSC were stained with MitoTracker Far Red (red) and iTreg were co-cultured for 48-72 h. iTregs were stained by CD4 antibody and analyzed. (c) Annexin V FACS analysis of MSC viability after cytochalasin B treatment. MSCs were gated as CD4 negative population. (d) FOXP3 expression in iTregs was measured from mock vs cytochalasin B treated MSC co-culture expanded iTregs $(n=5-7)$. (e) Effect of ROS inhibitor treatment on mitochondrial transfer. MSC were pre-stained with CFSE and MitoTracker Red FM and then cultured with Hoechst stained iTreg for $24-36 \mathrm{~h}$. The ROS inhibitor antioxidant $N$-acetylcysteine (NAC) $200 \mu \mathrm{M}$ was added to MSC + iTreg culture for 24-36 h. Live cell images were collected by confocal microscopy. (a,e) Analysis of recorded images was performed using Zen software Blue edition, (2011; version 2.0.14283.302). https://www. zeiss.com/microscopy/us/products/microscope-software/zen.html (f) MSC exosome uptake by proliferating iTregs. MSC exosome were stained with MitoTracker Far Red (Hough et al., 2018). FOXP3 ${ }^{+}$iTregs were stimulated with plate bound CD3 $\left(1 \mu \mathrm{g} \mathrm{ml}^{-1}\right)$ for $72 \mathrm{~h}$. Data are representative of 2-3 independent experiments. ${ }^{\star} p<0.05,{ }^{* *} p<0.001,{ }^{* * *} p<0.0001$ paired t test. Scale bars: $10 \mu \mathrm{m}$. See also Supplementary Figs. S4 and S5.

like cells and fibroblasts also transfer mitochondria ${ }^{40}$. However, we did not observe mitochondrial transfer to iTregs from other cell types including $\mathrm{CD} 133^{+}$hematopoietic stem cells (HSC) or HUVEC cells (Supplementary Fig. 5a). Further, we observed MSCs did not transfer mitochondria to iTregs after induction of MSC mitochondrial dysfunction ${ }^{40}$ (Supplementary Fig. 5b). Taken together, these data support a key role for the transfer of mitochondria from MSC to proliferating iTreg in enhancing iTreg number and suppressive function during IL-2 driven ex vivo expansion.

CD39/CD73 signaling plays a critical role in MSC mt transfer and stabilization of iTreg suppressive function. Experiments were conducted to determine the mechanisms driving MSC mt transfer into proliferating iTregs. Treg express apyrases (CD39) and ecto-5'-nucleotidase (CD73) that contribute to inhibitory function by generating adenosine ${ }^{41,42}$. Additionally, CD73-generated adenosine induces cortical actin polymerization via adenosine A1 receptor (A1R) induction of a Rho GTPase CDC42-dependent conformational change of the actin-related proteins 2 and 3 (ARP2/3) actin polymerization complex member N-WASP ${ }^{43}$. This is noteworthy since we identified MSC mt transfer to UCB iTreg occurs via cytochalasin B-sensitive, actin-dependent transport via TNTs. To test whether CD73 contributes to MSC mt transfer, we added CD73 blocking Ab to MSC co-cultures and measured iTreg mt mass. mt mass was significantly reduced in proliferating iTregs after CD73 blocking Ab treatment (Fig. 5a). To confirm MSC transfer mitochondria to iTregs during ex vivo expansion, we generated MSC stably expressing mitochondrially targeted GFP. MSCs were transduced with mito-GFP lentivirus to generate stable mtGFP ${ }^{+}$MSCs (Supplementary Fig. 6a) and co-cultured with iTregs expanded in IL-2/ media. Significantly less $\mathrm{mtGFP}^{+}$iTregs were detected during 21 day co-culture with mito-GFP lentivirus transduced MSCs when CD73 signaling was blocked or TNT formation was inhibited (Fig. 5b and Supplementary 
a

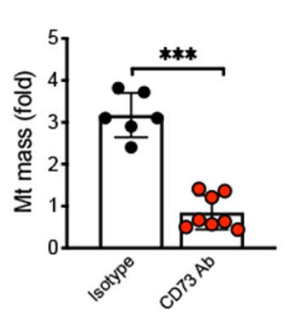

b

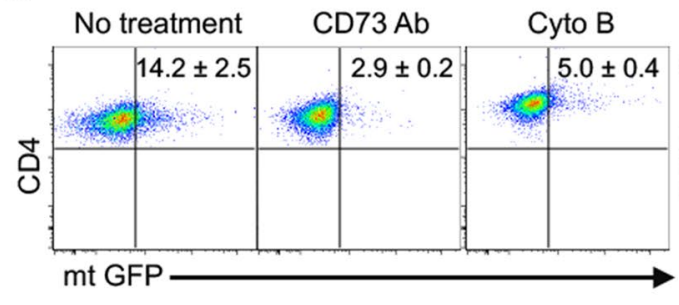

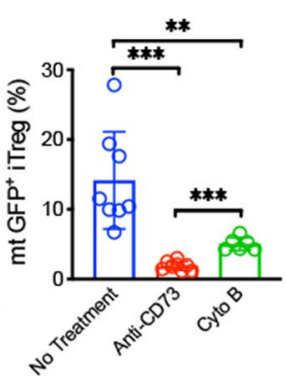

f

e$$
75
$$

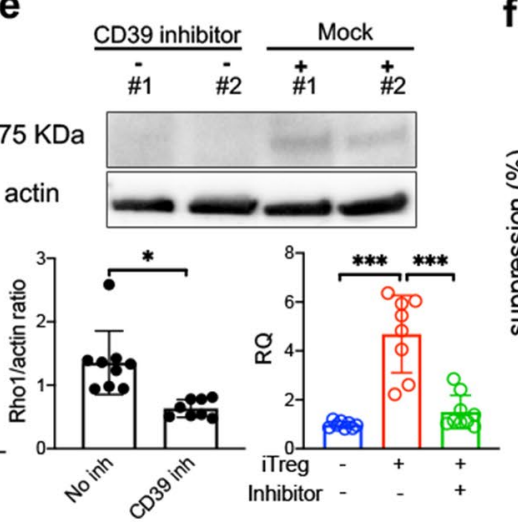

C

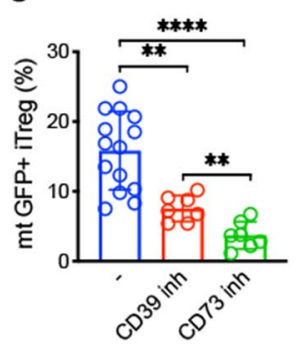

MSC co-culture/iTreg

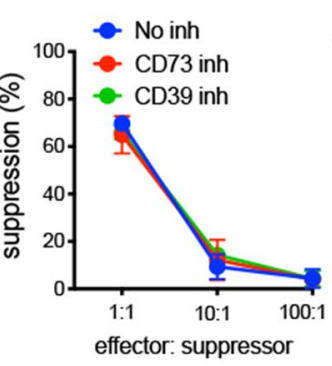

Figure 5. CD39/73 signaling induces MSC mitochondrial transfer to iTregs during IL-2 driven expansion. (a) Mitochondrial quantity was measured from IL-2/media or MSC co-culture expanded iTregs by RT-PCR $(n=6-8)$. Anti-CD73 antibody $\left(10 \mu \mathrm{g} \mathrm{ml}^{-1}\right)$ was added in MSC co-culture. Cells were collected at $72 \mathrm{~h}$ after mtGFP lentiviral transduced MSC co-culture. (b) Flow analysis of mtGFP ${ }^{+}$iTregs in anti-CD73 blocking and cytochalasin B treatment. Cells were stained with CD4 antibody in order to identify iTregs at $72 \mathrm{~h}$. (c) Analysis of $\mathrm{mtGFP}^{+} \mathrm{CD}^{+}$iTregs after incubation with CD39 $(100 \mathrm{nM})$ and CD73 $(100 \mathrm{nM})$ inhibitor treated MSC $(n=7-14)$. Representative flow plot, gated for CD4 $4^{+}$iTreg cells after MSC co-culture. (d) Protein and RNA expression of Mirol in MSC were measured after co-culture with iTreg. (e) After co-culture in the presence of CD39 inhibitor $(n=8-9)$. The image intensities for western blots were normalized to beta actin. Data are representative of 3-4 different experiments. (f) Effect of CD39 and CD73 inhibitors on suppressive functions of iTreg during MSC co-culture expansion. Inhibitor was added to iTreg and MSC co-culture. Data are representative of $n=3$ independent samples. ${ }^{*} p<0.05,{ }^{* *} p<0.01,{ }^{* * *} p<0.001,{ }^{* * *} p<0.0001$ paired t test. See also Supplementary Fig. S6.

Fig. 6b,c). As CD39 and CD73 calibrate purinergic signals delivered to immune cells through the conversion of ADP/ATP to AMP and AMP to adenosine, respectively ${ }^{44,45}$, we inhibited each pathway and observed reduced mtGFP ${ }^{+}$iTregs (Fig. 5c and Supplementary Fig. 6d).

As Mirol has been shown to be a key regulator in mitochondrial intracellular transport ${ }^{46}$, we sought to determine if Mirol played a role in MSC mt transfer to proliferating iTreg via TNT. We measured Mirol expression in MSC after co-culture with iTregs. Co-cultivation with proliferating iTreg was associated with significantly enhanced expression of Miro1 in MSC, including both protein and RNA levels (Fig. 5d). Further, the enhanced MSC expression of Mirol was inhibited by CD39 inhibition (Fig. 5e).

Since CD39/CD73 signaling play significant roles in mitochondrial transfer, we hypothesized these signaling pathways are critical to MSC-mediated enhancement of iTreg suppressive function. Inhibition of MSC mt into iTregs resulted in significantly reduced suppressive function vs. control (Fig. 5f, Supplementary Fig. 6d). These data indicate MSC enhance and stabilize iTreg FOXP3 expression and iTreg suppressive function via CD39/ CD73-induced mitochondrial transfer to proliferating iTregs during IL-2 driven expansion.

MSC mt transfer augments iTreg expression of FOXP3 stabilizing factors BACH2 and SENP3. BACH2 maintains stability and function of murine Treg ${ }^{15}$, 47 . We previously identified BACH2 is highly expressed in human UCB-derived iTreg and increases FOXP3 expression ${ }^{16}$. Additional studies have shown that SENP3 modulates BACH2 SUMOylation of and enhances iTreg stability in response to changing environmental conditions, particularly intracellular $\mathrm{ROS}^{17}$. We postulated mt transfer from MSC modulates BACH2/SENP3 expression and/or function, given our observations MSC mt transfer exerts a positive effect on iTreg Foxp3 stability and suppressive function ${ }^{17,48,49}$. We measured iTreg BACH2 and SENP3 protein expression, comparing MSC co-culture and standard IL-2/media suspension conditions during short-term (day 14 and 21) in vitro expansion. iTreg FOXP3, BACH2 and SENP3 expression was significantly increased in MSC co-culture conditions (Fig. 6a). We next examined iTreg BACH2 and SENP3 protein levels after cytochalasin B treatment to block mt transfer and observed loss of $\mathrm{BACH} 2$ protein expression (Fig. 6b). Cytochalasin $\mathrm{B}$ treatment had no 
a

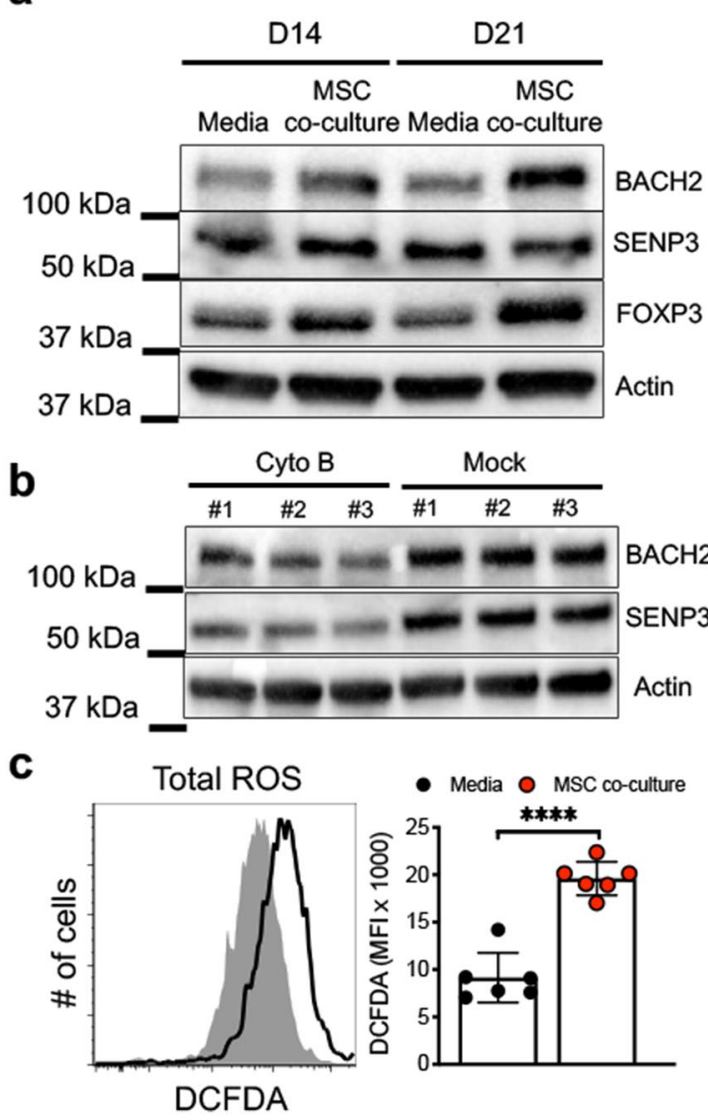

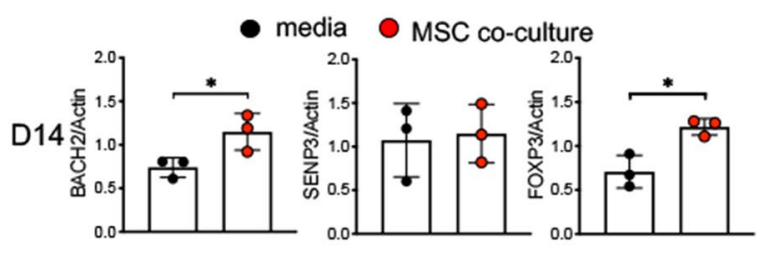
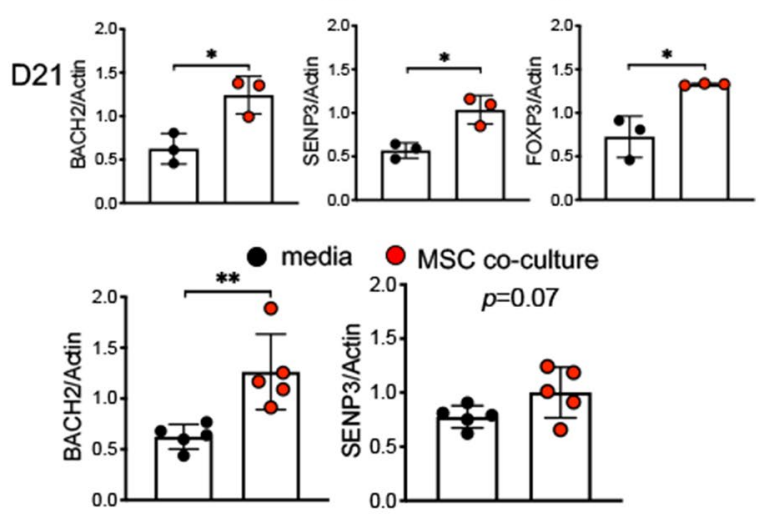

d
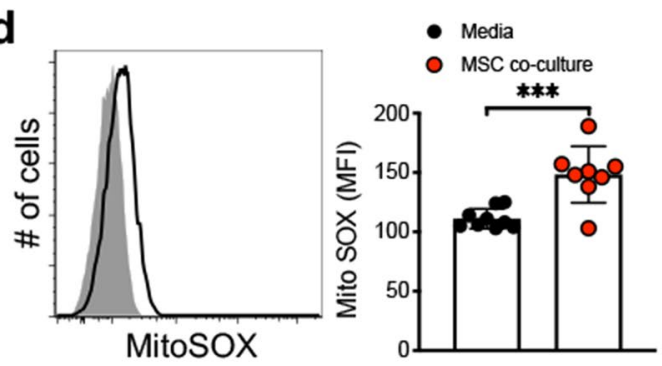

Figure 6. MSC co-culture induces iTreg BACH2 and SENP3 via ROS. (a) Expression of BACH2, SENP3, and FOXP3 on iTregs was measured by immunoblot assay. iTregs were expanded in media vs MSC co-culture and protein was isolated at indicated time. Data are representative of $n=3$ independent samples. (b) Analysis of BACH2 and SENP3 on iTregs after CD39 signaling inhibition. iTregs were expanded in media vs MSC co-culture and protein was isolated at indicated time. Expression was measured by immunoblot assay. (c,d) Expression of intracellular reactive oxygen species (ROS) was measured by flow cytometry in media vs MSC co-culture expanded iTregs (c) expression of mitochondrial ROS was measured by FACS analysis (d) at 21 days expansion. Data are representative of $n=6$ independent experiments. ${ }^{*} p<0.05,{ }^{* *} p<0.01,{ }^{* *} p<0.001$, ${ }^{* * * *} p<0.0001$ paired $t$ test. See also Supplementary Fig. S7.

significant effect on iTreg SENP3 protein expression, as SENP3 activation and/or function may be altered in this setting (Fig. 6b).

Previous studies have shown that mtROS induces $\mathrm{BACH} 2$ expression in $\mathrm{B}$ cells ${ }^{49}$. We measured $\mathrm{mtROS}$ expression in iTregs and found MSC co-culture induced higher expression of total and mtROS compared to standard IL-2/media suspension culture conditions (Fig. 6c,d). To investigate whether mtROS is a critical factor for induction of BACH2 and SENP3, we utilized a ROS inhibitor in iTreg/MSC co-cultures. Notably, iTreg expression of $\mathrm{BACH} 2$ and SENP3 in MSC co-cultures were significantly reduced in the presence of the ROS inhibitor (Supplementary Fig. 7). Overall, these data are consistent with MSC mt transfer enhancing iTreg BACH2 expression and subsequent enhanced FOXP3 expression and stability.

iTregs expanded on MSC feeder cells suppress effector CD4 and CD8 T cell responses in a xenogeneic GVHD NSG murine model. Inhibition of CD39 and CD73 signaling blocked MSC mt transfer to iTregs, and resulted in significantly reduced suppressive function vs. control (Fig. 5f). For assessment of the in vivo suppressive functions of iTreg expanded in MSC co-culture vs. iTreg expanded in standard IL-2/media suspension condition alone, iTregs were adoptively transferred into an NSG xenogeneic GVHD mouse model. iTregs expanded short-term (21 days) were injected 7 days after adult human PBMC were injected to induce GVHD. We observed enhanced survival and reduced lymphocyte infiltration into liver, skin, tongue and intestinal tissues in mice treated with iTregs from MSC co-cultures (Supplementary Fig. 8). RNA levels of FOXP3 and FOXP3 ${ }^{+} \mathrm{CD} 4 \mathrm{~T}$ cells in harvested spleen were significantly increased in mice treated with MSC co-cultured iTregs at 2 weeks post-GVHD induction (Fig. 7a,b). IFN $\gamma$ producing $\mathrm{CD} 8^{+}$and $\mathrm{CD} 4^{+} \mathrm{T}$ cells were dramatically reduced in spleen cells harvested from mice treated with MSC co-cultured iTregs (Fig. 7d). Consistent with the cytokine staining results, serum and ex vivo levels of pro-inflammatory cytokines IFN $\gamma$ and TNF were significantly reduced in animals treated with MSC co-cultured iTregs (Supplementary Fig. 9a,b). 
a
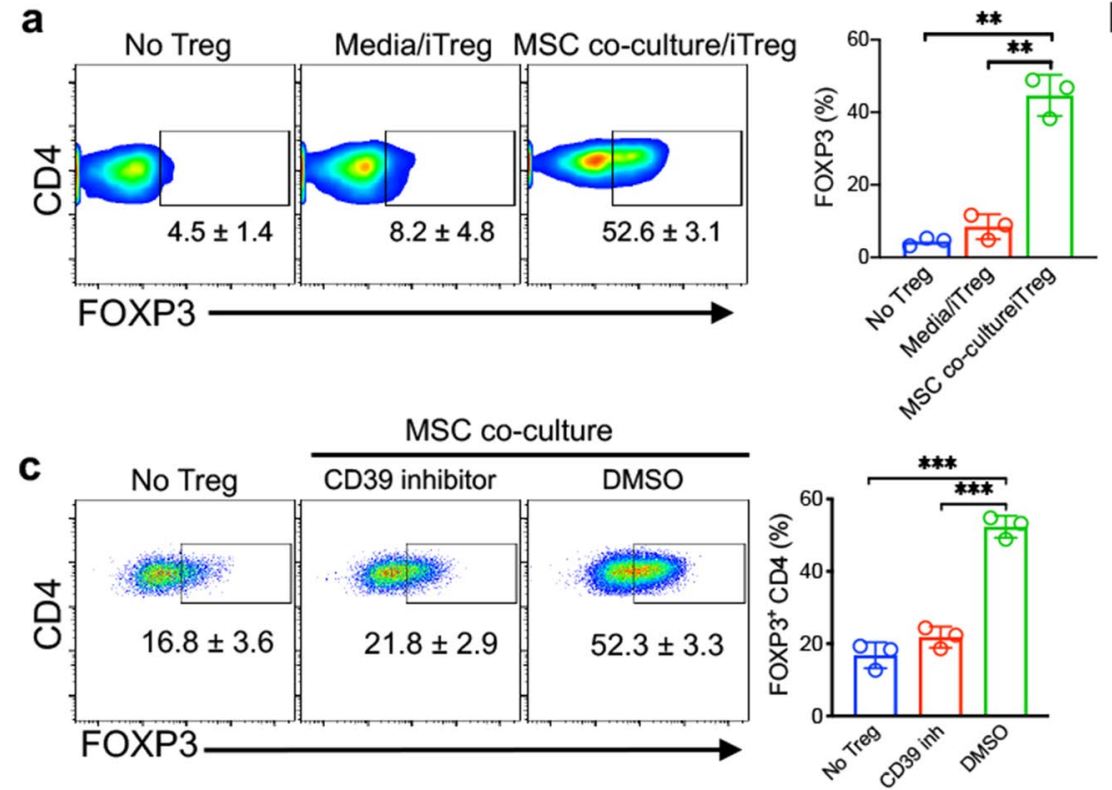

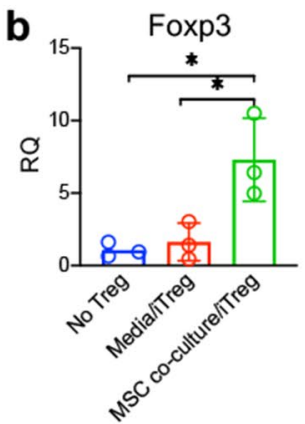

MSC co-culture expansion
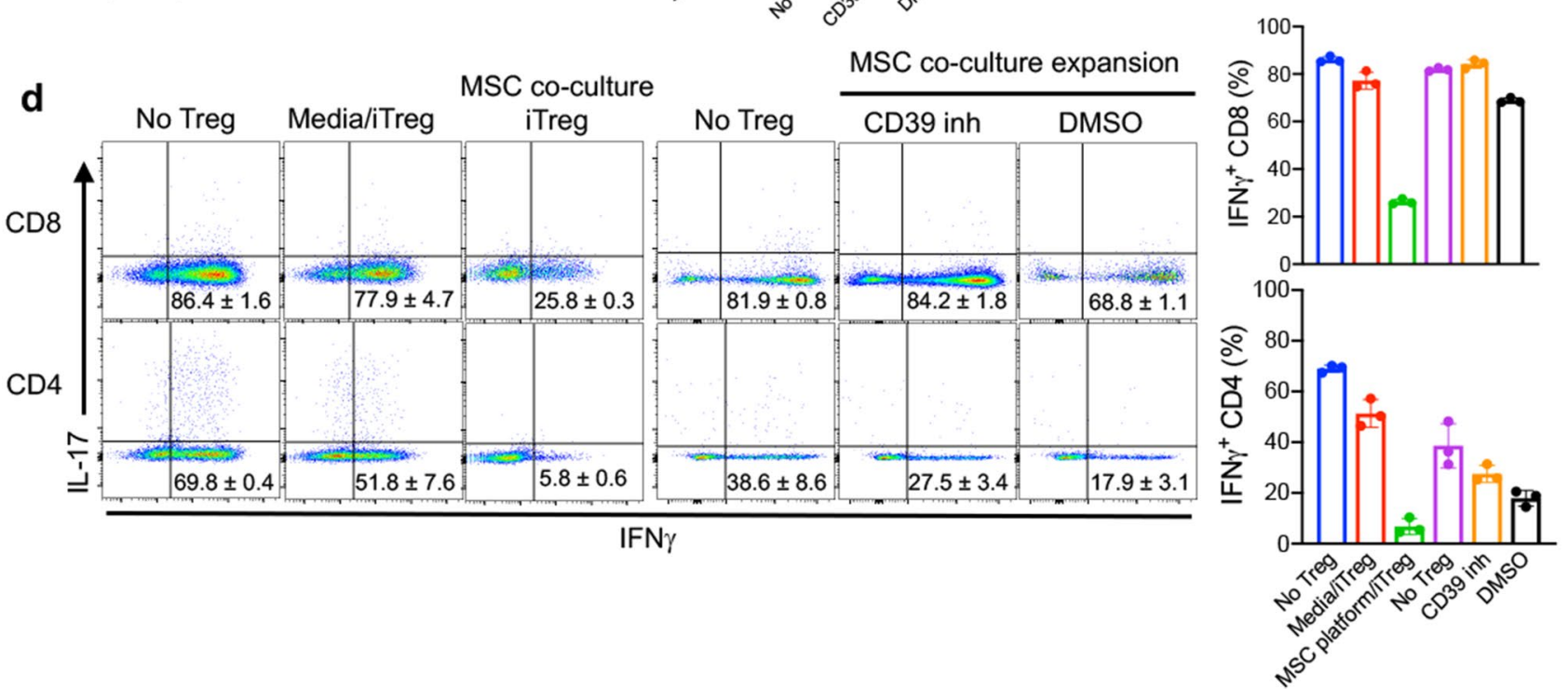

Figure 7. MSC co-culture expanded iTregs suppress effector T cell response in GVHD inflammation. (a) $\mathrm{FOXP}^{+} \mathrm{CD} 4 \mathrm{~T}$ cells were measured from no iTreg, media expanded iTreg, and MSC co-culture expanded iTreg treated mice. NSG mice received $10^{7} \mathrm{PBL}$ and iTregs were injected at 7 days after PBL injection. Spleen cells were collected at day 14 after PBL injection. (b) FOXP3 expression, as quantified by RT-PCR, in the spleen cells. Measurement of FOXP3 ${ }^{+} \mathrm{CD} 4 \mathrm{~T}$ cells in iTreg-treated mice. iTregs were expanded in MSC co-culture with CD39 inhibitor vs control. 10 days after expansion, iTreg cells were injected into PBL-treated NSG mice. Spleen cells were collected at day 14 after PBL injection. Expression of IFN $\gamma$ and IL-17 was measured by flow cytometry in $\mathrm{CD} 4 \mathrm{~T}$ cells. (d) Measurement of IFN $\gamma$ producing $\mathrm{CD} 4$ and $\mathrm{CD} 8 \mathrm{~T}$ cells was performed by flow cytometric analysis. Data are representative of $n=3-4$ independent samples. ${ }^{*} p<0.05,{ }^{* *} p<0.01,{ }^{* * *} p<0.001$ paired $\mathrm{t}$ test. See also Supplementary Figs. S8 and S9.

To explore the effect of MSC $\mathrm{mt}$ transfer on iTreg phenotype and function in vivo, we co-cultured iTregs with MSC in the presence of a CD39 inhibitor ${ }^{50,51}$. CD39 inhibitor treatment reduced the percentage of FOXP $3^{+}$iTreg in PBL induced GVHD NSG mice (Fig. 7c). IFN $\gamma$ producing $\mathrm{CD}^{+}$and $\mathrm{CD}^{+} \mathrm{T}$ cells were significantly increased in mice injected with CD39 inhibitor-treated iTreg (Fig. 7d). IFN $\gamma$ and TNF were also significantly increased in the serum of these mice (Supplementary Fig. 9c) and ex vivo (Supplementary Fig. 9d). Collectively, these results reveal suppressive iTregs are maintained by MSC mt transfer and can suppress effector T cell immune responses against human inflammatory xeno-immune disease in vivo.

\section{Discussion}

Intrinsic defects in Tregs as observed in autoimmune disease may hamper the success of autologous tTreg adoptive cell therapies ${ }^{52-54}$. Previous studies have shown ex vivo-expanded, partially HLA-matched tTregs from allogeneic UCB sourcing are well tolerated in humans ${ }^{11}$. UCB-derived iTregs exhibit enhanced stability and 
suppressive function compared to adult blood-derived iTregs in large part due to enhanced expression of the FOXP3-stabilizing transcription factor $\mathrm{BACH} 2^{16}$. Nevertheless, a major obstacle to the clinical implementation of iTregs is concern over the instability of iTreg FOXP3 expression and loss of suppressor function upon transfer to an inflammatory environment ${ }^{55}$. The results reported in this study demonstrate that MSC co-culture provides enhanced and sustained FOXP3 expression and iTreg suppressive function in inflammatory in vitro and in vivo environments. Importantly, our study provides insight into normal physiologic mechanisms to sustain human proliferating T-cells including mt transfer from adjacent MSC.

Co-culture of iTregs with MSC feeder cells significantly improved the number and suppressive function of iTreg during 21 day IL-2 based ex vivo expansion. MSC co-culture increased iTreg expression of CD25, CTLA4 , and ICOS, while expression of LAG-3 and TIM-3, associated with exhaustion of activated T cells ${ }^{56-62}$, was decreased. We observed higher proportions of CD62 $\mathrm{L}^{+}$and absolute numbers of CD45RA ${ }^{+}$iTreg cells after 21 day IL-2 expansion co-culture on MSC feeder cells. Inflammatory environments exert rapid loss of FOXP3 expression and reduced suppressive activity of iTregs ${ }^{63}$. Our data show significantly enhanced suppressive function, including inhibition of effector cell activation and maintained FOXP3 stability in MSC co-culture expanded iTregs exposed to inflammatory conditions in vitro and in vivo. It will be important in the future to examine whether epigenetic modification of the Treg-specific demethylated region in the FOXP3 gene occurs, in order to gain further insights into the mechanisms of MSC-enhanced iTreg stability ${ }^{64}$.

Although prior studies point to MSC enhancement of Treg function to be primarily via paracrine mechanisms ${ }^{65-67}$, our study shows MSC support of iTreg FOXP3 expression and sustained suppressive function requires cell-cell contact $\mathrm{mt}$ transfer. Recent work showed umbilical cord-derived MSC mt can promote adult PBMC CD4 T cell differentiation into iTreg ${ }^{68}$. Intriguingly, purified MSC mt could recapitulate the effects of MSC $\mathrm{mt}$ transfer to promote Treg differentiation. However, this study did not examine stabilization of iTreg FOXP3 expression and maintenance of naïve phenotype mediated by MSC $\mathrm{mt}$ transfer during ex vivo expansion. Our findings suggest direct cell-cell contact and $\mathrm{mt}$ transfer via TNTs play an important role in stabilization of iTreg FOXP3 expression, maintenance of naïve phenotype, and maintained iTreg suppressive function in adverse inflammatory conditions. MSC co-culture was also observed to support maintenance of tTreg phenotype. Notably however, the proportion of FOXP $3^{+}$tTregs was less sustained compared to iTregs during expansion (57\% vs $96 \%$, respectively at Day 21 in MSC co-culture) potentially reflecting the presence of contaminating effector cells and the more stable phenotype of iTregs generally.

Several mechanisms have been proposed to explain how mt may promote Treg formation. For instance, it was proposed retrograde mitochondria to nuclear signaling alters the expression of nuclear genes involved in Treg phenotype via epigenetic mechanisms ${ }^{68}$. However, prior to our studies, the cellular and molecular mechanisms driving MSC mt transfer to proliferating cells and signaling pathways leading to altered phenotype of recipient cells have not been elucidated. Our studies identify iTreg CD39/CD73 signaling drives MSC mt transfer and further mt transfer results in augmented iTreg BACH2 and SENP3 expression. BACH2 regulates human UCB iTreg development via direct transcriptional activity at the FOXP3 promoter, and by suppressing TCR induced gene transcription ${ }^{16,69}$. SENP3 is a SUMO specific protease known to maintain Treg stability ${ }^{17}$. SENP3 activity can be regulated by a variety of cell stressors including but not limited to ROS ${ }^{70}$. In addition, SENP3 has been linked to mitochondrial signaling mechanisms and function. SENP3 can deSUMOylate and modulate the localization and function of Drp1, a key regulator of mitochondrial fission in neurons. Prior studies have also identified a role for mitochondrial regulation of $\mathrm{BACH} 2$ function and cell fate decisions in $\mathrm{B}$ cells ${ }^{49}$. Thus, our findings suggest a potential mechanism whereby mitochondrial transfer stabilizes iTreg suppressor function via altered expression of SENP3 and BACH2.

CD39 and CD73 together play strategic roles in immune responses ${ }^{44,45}$. CD39 and CD73 degrade extracellular ATP to yield AMP and anti-inflammatory adenosine ${ }^{71}$. CD73-/- mice show enhanced antitumor immunity ${ }^{72}$ and more severe gastritis compared to functional CD73 controls, and adoptive injection of WT Tregs reversed these immune responses ${ }^{42}$. Prior studies ${ }^{73,74}$ identify CD73 signaling on Tregs is critical to maintain Treg suppressive function. Our study identifies MSC mt transfer to iTregs contributes to enhanced iTreg phenotypic stabilization. In addition, the signaling mechanisms involved in $\mathrm{mt}$ transfer to Tregs have not been previously examined. We identify CD39 and CD73 signaling mediates MSC mitochondrial transfer to proliferating iTregs during IL-2 driven ex vivo expansion. Further studies on CD39 and CD73 mediated mt transfer may elucidate mechanisms that may be exploited for Treg functional stabilization.

MSCs mt transfer has been shown in several cell types, including proliferating acute leukemia blasts in the marrow microenvironment ${ }^{39}$. Molecular pathways of mitochondrial transfer have been identified, including the mitochondrial Rho-GTPase $1 \mathrm{Miro}^{40}$. Mirol regulates intercellular mt transfer by attaching mitochondria to KLF5 the kinesin motor protein ${ }^{75,76}$ and has been implicated in enhanced injured cell recovery ${ }^{40}$. In one study, injured astrocytes induced increased levels of Miro1 expression in MSC and this was correlated with $\mathrm{mt}$ transfer ${ }^{20}$. However, MSC mt transfer to Treg cells and the cellular and molecular mechanisms underlying $\mathrm{mt}$ transfer has not been previously identified. Our data show mitochondrial metabolic function through CD39/ CD73 signaling, in proliferating iTregs induced Mirol expression in MSC during co-culture ex vivo. MSC mt transfer occurs via TNT rather than exosome mediated transfer. These findings provide impetus to study the cell signaling mechanisms in proliferating iTreg serving to induce MSC Mirol expression and facilitate TNT formation for $m t$ transfer.

Mitochondria are an important source of $\operatorname{ROS}^{77}$ and regulate cell cycle, modulate signal transduction ${ }^{78,79}$, and are critical for cancer cell tumorigenicity ${ }^{80}$. This is particularly relevant considering ROS is a major driving force for $\mathrm{mt}$ transfer via TNTs from bone marrow stromal cells to leukemic blasts ${ }^{39}$. Further work demonstrated $\mathrm{mt}$ metabolism plays a critical role in T cell activation. Thus, future studies are warranted to investigate whether the transfer of MSC mt modulates these metabolic signaling pathways in proliferating Treg. Our observations strongly indicate increased mt quantity in iTregs was derived from MSCs via TNT transfer. However, ROS was 
not a critical mechanism driving this process. Nonetheless, cytochalasin B treatment led to diminished number of FOXP3 ${ }^{+}$iTregs, suggesting TNT transfer of $\mathrm{mt}$ from MSC play an important role in maintenance of FOXP3 ${ }^{+}$ iTreg stability.

Regardless of cell type, $\mathrm{mt}$ are now recognized to play roles extending well beyond the production of energy. $\mathrm{T}$ cells undergoing changes in cellular metabolism during activation is a central idea emerging from many recent studies $^{81}$. In contrast to quiescent $\mathrm{T}$ cells, with limited metabolic needs mainly encompassing cellular trafficking and housekeeping functions, actively proliferating cells must generate additional ATP for functions including the generation of intermediates required in various biosynthetic pathways and signaling molecules for anabolic metabolism. Future work is needed to identify how signaling mechanisms can be modulated to modulate mt transfer. Importantly, several lines of evidence support mt transfer is a key mechanism of MSC supportive function. Our studies identify iTreg mt membrane potential is enhanced by culture over MSC feeder cells, and further pharmacologic inhibition of mt transfer nearly completely negates the benefit of MSCs on iTreg FOXP3 expression and suppressive function.

In summary, our findings highlight the importance of mt function in maintaining iTreg stability in MSC coculture expanded iTreg and provide insight into cellular and molecular mechanisms driving MSC mt transfer to proliferating iTreg. This process inhibits differentiation, cell senescence, maintains robust FOXP3 expression, as well as suppressive function despite an adverse inflammatory milieu in vitro and in vivo. Although several factors modulate iTreg stability and function in disease models, our results show the mt BACH2 pathway is a prominent mechanism of MSC-enhanced iTreg stability and function. Additionally our work highlights the important role played by CD73-regulated TNT formation that serves to facilitate $\mathrm{mt}$ transfer into proliferating iTreg during expansion.

\section{Methods}

Mice. NSG mice were ordered from Case Western Reserve University (CWRU) a thymic facility. Mice were maintained in the SPF animal facility at CWRU. Mice were ear-punched, weighed, placed in 12-pie irradiator holder and exposed to 240 rads from Gammacel ${ }^{137}$ Cs source.

Human bone marrow MSC culture. De-identified bone marrow samples were obtained from human BM aspirate from the CWRU Biorepository. Briefly, marrow mononuclear cells were resuspended at a concentration of $2 \times 10^{6}$ cells $/ \mathrm{ml}$ in completed IMDM (GIBCO BRL Life Technologies, Grand Island, NY, USA) medium. Media contained 20\% heat-inactivated human serum albumin (Gemini Bio-product, Sacramento, CA) with 1\% penicillin and streptomycin (Gibco), $40 \mathrm{ng} / \mathrm{ml}$ basic fibroblastic growth factor (Sigma). Cells were cultured in $175 \mathrm{~cm}^{2}$ flasks and incubated at $37{ }^{\circ} \mathrm{C} 5 \% \mathrm{CO}_{2}$ in a humidified incubator for 2-4 weeks until a confluent layer was formed. BM-MSCs were characterized by analyzing the expression of CD markers (CD11b, CD19, CD34, CD44, CD45, CD73, CD90, CD105, and HLA-DR; BD Stemflow hMSC Analysis Kit; BD Biosciences, San Jose, CA, USA) by flow cytometry. MSCs between passages 3-5 were used in experiments.

Human UCB and adult peripheral blood samples. Acquisition of de-identified volunteer young adult peripheral blood and UCB were obtained with written informed consent per Case Western Reserve University/Cleveland Cord Blood Center IRB-approved protocols. All methods in these studies were carried out in accordance with relevant guidelines and regulations. Mononuclear cells were isolated by Ficoll-Paque PLUS (GE Healthcare Life Sciences, Piscataway, NJ) density gradient centrifugation with SepMate-50 tubes (STEMCELL Technologies, Vancouver, BC, Canada).

FOXP3 $3^{+}$iTreg generation and ex vivo expansion. UCB derived FOXP $3^{+}$iTregs cells were generated as previous described ${ }^{82}$. Ex vivo expansion of FOXP3 ${ }^{+}$iTregs was performed with $\mathrm{CD}^{2} 5^{+}$cells MACS purified from day 4 TGF $\beta$-induced UCB iTreg. On Day 4 of TGF- $\beta$ conditioning, CD $25+$ iTregs were MACS purified, rested for $48 \mathrm{~h}$, and on Day $6,5 \times 10^{5}$ purified cells were split into expansion cultures with either media containing 100 $\mathrm{U} / \mathrm{ml}$ IL-2 in suspension or identical media/IL-2 over MSC feeder cells. Cultures were fed every other day with fresh media/IL-2 and MSC during short-term culture (21-28 days). No re-stimulation was performed during the 21-28 day expansion following the initial 4 day stimulation consistent with a previously described UCB Treg expansion protocol ${ }^{83}$. For isolation of UCB-derived tTregs, EasySep Human CD4 + CD127lowCD25 + Regulatory T Cell Isolation Kit (Stem Cell Technologies) was used. tTregs were maintained under identical conditions as iTregs. Absolute number of FOXP3 ${ }^{+}$iTreg cells were calculated from the percentage of $\mathrm{CD} 4^{+}$cells based on the total viable cell count obtained by trypan blue dye exclusion at each indicated time point. For iTreg stability assays, harvested iTregs were stimulated with CD3 and CD28 antibodies $\left(1 \mu \mathrm{g} \mathrm{ml}^{-1}\right)$ with IFN $\gamma\left(10 \mu \mathrm{g} \mathrm{ml}{ }^{-1}\right)$, IL-6 $\left(10 \mu \mathrm{g} \mathrm{ml}^{-1}\right)$, and TNF $\left(10 \mu \mathrm{g} \mathrm{ml}^{-1}\right)$ at concentration of $5 \times 10^{5} \mathrm{cells} \mathrm{ml}^{-1}$ for $72 \mathrm{~h}$ and analyzed.

Flow cytometry analysis. Cells were harvested on days 7, 14, and 21 of iTreg expansion, and were surface stained with antibodies against CD4-FITC, PECy7 (RPA-T4), CD8-FITC (RPA-T8), CD25-PE (M-A251), CD45RA-PE CF594 (HI100), CD45RO-PE (UCHL1), CD62L-BB515 (SK11), CD223 (LAG-3)-BV421 (T47530), CD304 (NRP-1)-Alexa Fluor 647 (U21-1283), CD278 (ICOS)-PECy7 (398.4A), CD279 (PD-1)-PE (EH12.1), CD366 (Tim-3)-Alexa Fluor 647 (7D3) (BD Bioscience, San Diego, CA). For CTLA-4 and FOXP3 cytoplasmic staining, APC anti-CTLA-4 (BNI3, BD pharmingen) and PE anti-FOXP3 (3G3, Miltenyi, Auburn, CA, USA) antibodies were utilized. For intracellular staining, cells were fixed after surface staining and permeabilized with Fixation/Permeabilization kit according to the manufacturer's protocol (Miltenyi). A Fortessa instrument (BD Biosciences) was used for data acquisition and the data was analyzed using FlowJo software (Tree Star, Inc., Ashland, OR). To measure intracellular cytokines, cells were stimulated for $4 \mathrm{~h}$ with PMA (10 
$\mathrm{ng} / \mathrm{ml}$; Sigma-Aldrich) and Ionomycin $(1 \mu \mathrm{M})$ in the presence of $2 \mu \mathrm{M}$ Monensin (Calbiochem; San Diego, CA) during the last $2 \mathrm{~h}$ of incubation and fixed with $4 \%$ paraformaldehyde, permeabilized, and stained with fluorochrome conjugated antibodies.

Suppression assay. In vitro suppression assays were performed as previously described ${ }^{82}$. At 3 weeks of expansion, CD25 $5^{\text {int }}$ FACS sorted iTregs were used for suppression assays. Responder naïve CD4 ${ }^{+} \mathrm{T}$-cells and Mitomycin C treated T-cell depleted PBMC were prepared from a healthy donor. CFSE labeled naïve CD4 ${ }^{+}$ T-cells were plated at a 1:1 ratio with Mitomycin C treated T-depleted PBMC and varying concentrations of UCB iTreg. Soluble anti-CD3 $(2 \mu \mathrm{g} / \mathrm{ml}$ ) (clone HIT3a, BD Bioscience) mAb was added. Suppression was measured by cell division index by FACS.

Measurement of mt transfer. For mt and cell labeling, MitoTracker Green, MitoTracker Red FM, and CellTrace Far Red were purchased from Thermo Fisher Scientific. MSCs were incubated in MitoTracker Green $(200 \mathrm{nM})$ or MitoTracker Red FM (500 nM) for 45 min and washed 3 times with pre-warmed media and iTreg cells were incubated in CellTrace Far Red and stained according to the manufacturer's instructions. MSC and iTreg were then cocultured for $24-36 \mathrm{~h}$ and then assessed via flow cytometry or imaged at indicated time periods on $35 \mathrm{~mm}$ \#1.5 glass bottomed petri dish (Cellvis; Mountain View, CA) using a Zeiss Axiovert microscope equipped with Apotome. 2 using the $63 \times$ oil immersion objective. Analysis of recorded images was performed using Zen 2012 software.

Quantitative real-time polymerase chain reaction. Total RNA was extracted from cells using GeneJet RNA Purification Kit (Thermo Scientific, Waltham, MA) and quantified. cDNA was subsequently synthesized using High Capacity cDNA Reverse Transcription Kits (ThermoFisher). Real time quantitative PCR was performed using gene-specific primers and probe sets (Applied Biosystem, Foster City, CA) and a QuantStudio 3 RT-PCR instrument (Applied Biosystems). The qRT-PCR runs were performed in triplicate to quantify expression levels for each gene using Taq-man assays per manufacturer's instructions.

ROS measurement. To measure mt ROS production, $1 \times 10^{6}$ cells were incubated in complete media with $5 \mu \mathrm{M}$ MitoSox (Invtirogen, Carlsbad, CA) for $30 \mathrm{~min}$ at $37^{\circ} \mathrm{C}$. Cells were washed with pre-warmed media and FACS buffer. Cells were stained with relevant surface Abs for FACS analysis. To measure total ROS, $1 \mu \mathrm{M}$ $2^{\prime}, 7^{\prime}$-dichlorofluorescin diacetate (DCFDA) was used. Data were acquired on a Fortessa (BD Bioscience) and analyzed using FlowJo (Tree Star).

Mitochondria mass quantification. To quantify mt DNA copy number, we used Terra qPCR direct polymerase mix and Human Mitochondria DNA (mtDNA) monitoring primer set (Takara Bio USA) according to the manufacturer's instructions.

Western blot analysis. Collected cells were lysed using radio-immune precipitation assay (RIPA) buffer and protein was quantified using the Bradford assay (BioRad, Hercules, CA), with $40 \mu \mathrm{g}$ of protein separated by $4-20 \%$ SDS-PAGE (BioRad). Proteins were electro-transferred onto Immobilon membrane (Millipore) and were probed with polyclonal rabbit anti-BACH2 (D3T3G; Cell signaling technology, Beverly, MA) anti-SENP3 (D20A10; Cell signaling technology) antibody, monoclonal rabbit anti-FOXP3 (D25D4; Cell signaling technology), and monoclonal mouse/human/rat anti- $\beta$-actin antibody (R\&D systems, Minneapolis, MN). Applicable HRP-conjugated secondary antibodies were used with ECL chemi-luminescence to observe relevant bands using ChemiDoc XRS + image system (BioRad). The band intensity was quantified using Image J.

Cytoplasmic and nuclear protein isolation. Cytoplasmic and nuclear fractions were isolated from $\sim 10-20 \times 10^{6}$ Treg cells from IL-2/media only cultures or Tregs from IL-2/media MSC co-cultures using the NE-PER Nuclear and Cytoplasmic extraction reagents kit (Invitrogen 78833) according to the manufacturer's instructions. In brief, Treg cells were removed from co-culture, washed with PBS, and ice cold CER1 reagent was added, and samples were pelleted by centrifugation. Samples were vortexed for $15 \mathrm{~s}$ and incubated on ice for $10 \mathrm{~min}$. Ice cold CERII reagent was added and samples were vortexed for an additional $5 \mathrm{~s}$ and incubated on ice for $1 \mathrm{~min}$. After additional $5 \mathrm{~s}$ vortex, samples were centrifuged for $5 \mathrm{~min}$ at maximum speed. The supernatant containing the cytoplasmic extract was transferred to a new tube. The insoluble pellet fraction containing the nuclei was suspended in ice-cold NER and vortexed for $15 \mathrm{~s}$ every $10 \mathrm{~min}$ for $40 \mathrm{~min}$. Nuclear fraction was centrifuged at maximum speed for $10 \mathrm{~min}$ and supernatant containing the nuclear extract was transferred to a new tube. Isolated fractions were kept on ice. Protein concentration was determined by Bradford assay and equivalent amount of total protein for each sample (5-10 $\mu \mathrm{g}$ nuclear fraction, 20-40 $\mu \mathrm{g}$ cytoplasmic fraction) was loaded onto a 4-20\% SDS-PAGE gel and processed for analysis of BACH2 and SENP3 expression by western blotting as described above.

5-Bromo-2'-deoxyuridine (BrdU) assay. $10 \mu \mathrm{M} \mathrm{BrdU}$ was added to media or MSC co-culture iTreg expansion culture well during the last $48 \mathrm{~h}$. After $2 \mathrm{~h}$ incorporation, iTreg cells were harvested and BrdU staining was performed per manufacturer's instructions (Invitrogen). Cells were stained with CD4 to exclude non iTregs.

pLV-mitoGFP Lentivirus production and transduction. Lentiviral plasmids pLV-eGFP and pLVmitoGFP were gifts from Pantelis Tsoulfas (Addgene plasmids \# 36083 and 44385) ${ }^{84,85}$. Lentiviral plasmids were 
isolated from Stbl3 cells by minipreps (Qiagen) of $5 \mathrm{~mL}$ overnight cultures established from single colonies. Lentivirus was produced in HEK293T cells. Transduction experiments were performed by spinoculation transduction method as described ${ }^{86}$.

ELISA assay. Levels of IFN $\gamma$ and TNF were detected from serum and culture supernatant. IFN $\gamma$ and TNF were determined by ELISA (R\&D systems), according to the manufacturer's instructions.

GVHD model and tissue histology. For establishment of an acute GVHD model, NSG mice received cord blood CD $34^{+}$cells. 5-6 weeks later, mice were injected with adult PBL $(1 \times 10 \mathrm{e} 7)$. GVHD severity was assessed by an established scoring system based on weight loss, posture, activity, fur texture and skin texture ${ }^{87}$. Pathologic evaluation was performed for organs targeted by acute GVHD, including liver, skin, tongue and intestine $^{88}$. Tissues were collected and hematoxylin/eosin (H\&E) stained at day 21 after injection of PBL. Intestines were washed thoroughly prior to sample collection. All samples were fixed using $10 \%$ neutral-buffered

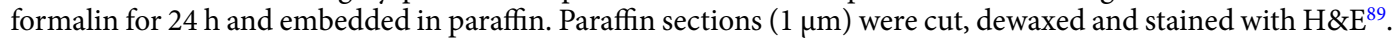

Statistical analysis. Statistical comparative analyses were performed using the Paired $t$-test (Prism 7 software-GraphPad, San Diego, CA). Multiple comparisons analysis was performed using the Friedman test. Data are presented as the mean \pm standard deviation, SD. A $p$ value of $<0.05$ was considered significant. ${ }^{\star} p<0.05$, ${ }^{* *} p<0.01,{ }^{* * *} p<0.001$.

Study approval. This study was designed to determine whether MSCs can enhance the stability and suppressive capacity of iTregs during ex vivo expansion. MSC were obtained from healthy donor derived bone marrow and umbilical cord blood from at least 3 different donors to confirm our observation of MSC mitochondrial transfer mediated stabilization of iTregs. The study was carried out in accordance with the approved guidelines and regulations. De-identified human cells tested in this study were approved by Cleveland Clinic Foundation for volunteer umbilical cord blood collection with written informed consent from donating mothers. In addition, the collection of normal adult peripheral blood from de-identified human donors was approved by the Case Western Reserve University IRB for the Case Comprehensive Cancer Center Hematopoietic Stem Cell Facility (CASE 12Z05). For GVHD studies, iTregs expanded in either media/IL-2 suspension culture condition or media/IL-2 over MSC feeder cells, were harvested and injections were performed by a second investigator (DA) who was blinded to the identity of each sample. All animal experiments and methods were performed in accordance with the relevant guidelines and regulations approved by the Institutional Animal Care and Use Committee of Case Western Reserve University. In addition, mice studies were carried out in compliance with the ARRIVE guidelines. Mice were randomized on the basis of their age, sex and weight. Numbers of mice were chosen based on magnitude of observed effects and variability in the results and are indicated in figure legends.

\section{Data availability}

All data generated or analyzed during this study are included in this published article and its Supplementary Information files.

Received: 27 August 2020; Accepted: 6 May 2021

Published online: 21 May 2021

\section{References}

1. Chen, W. et al. Conversion of peripheral CD4+CD25- naive T cells to CD4+CD25+ regulatory T cells by TGF-beta induction of transcription factor Foxp3. J. Exp. Med. 198, 1875-1886. https://doi.org/10.1084/jem.20030152 (2003).

2. Davidson, T. S., DiPaolo, R. J., Andersson, J. \& Shevach, E. M. Cutting Edge: IL-2 is essential for TGF-beta-mediated induction of Foxp3+ T regulatory cells. J. Immunol. 178, 4022-4026 (2007).

3. Schmitt, E. G. \& Williams, C. B. Generation and function of induced regulatory T cells. Front. Immunol. 4, 152. https://doi.org/ 10.3389/fimmu.2013.00152 (2013).

4. Kanamori, M., Nakatsukasa, H., Okada, M., Lu, Q. \& Yoshimura, A. Induced regulatory T cells: Their development, stability, and applications. Trends Immunol. 37, 803-811. https://doi.org/10.1016/j.it.2016.08.012 (2016).

5. Bluestone, J. A., Trotta, E. \& Xu, D. The therapeutic potential of regulatory T cells for the treatment of autoimmune disease. Expert Opin. Ther. Targets 19, 1091-1103. https://doi.org/10.1517/14728222.2015.1037282 (2015).

6. Le Texier, L. et al. Autophagy-dependent regulatory T cells are critical for the control of graft-versus-host disease. JCI Insight $\mathbf{1}$, e86850. https://doi.org/10.1172/jci.insight.86850 (2016).

7. Baecher-Allan, C., Brown, J. A., Freeman, G. J. \& Hafler, D. A. CD4+CD25high regulatory cells in human peripheral blood. J. Immunol. 167, 1245-1253. https://doi.org/10.4049/jimmunol.167.3.1245 (2001).

8. Huehn, J., Polansky, J. K. \& Hamann, A. Epigenetic control of FOXP3 expression: The key to a stable regulatory T-cell lineage?. Nat. Rev. Immunol. 9, 83-89. https://doi.org/10.1038/nri2474 (2009).

9. Trzonkowski, P., Szarynska, M., Mysliwska, J. \& Mysliwski, A. Ex vivo expansion of CD4(+)CD25(+) T regulatory cells for immunosuppressive therapy. Cytometry A 75, 175-188. https://doi.org/10.1002/cyto.a.20659 (2009).

10. Long, S. A. et al. Defects in IL-2R signaling contribute to diminished maintenance of FOXP3 expression in CD4(+)CD25(+) regulatory T-cells of type 1 diabetic subjects. Diabetes 59, 407-415. https://doi.org/10.2337/db09-0694 (2010).

11. Brunstein, C. G. et al. Infusion of ex vivo expanded T regulatory cells in adults transplanted with umbilical cord blood: Safety profile and detection kinetics. Blood 117, 1061-1070. https://doi.org/10.1182/blood-2010-07-293795 (2011).

12. Tone, Y. et al. Smad3 and NFAT cooperate to induce Foxp3 expression through its enhancer. Nat. Immunol. 9, 194-202. https:// doi.org/10.1038/ni1549 (2008).

13. Rauch, K. S. et al. Id 3 maintains Foxp3 expression in regulatory T cells by controlling a transcriptional network of E47, Spi-B, and SOCS3. Cell Rep. 17, 2827-2836. https://doi.org/10.1016/j.celrep.2016.11.045 (2016).

14. Roychoudhuri, R. et al. The transcription factor BACH2 promotes tumor immunosuppression. J. Clin. Investig. 126, 599-604. https://doi.org/10.1172/JCI82884 (2016). 
15. Roychoudhuri, R. et al. $\mathrm{BACH} 2$ represses effector programs to stabilize T(reg)-mediated immune homeostasis. Nature 498, 506510. https://doi.org/10.1038/nature12199 (2013).

16. Do, J. S. et al. Foxp3 expression in induced T regulatory cells derived from human umbilical cord blood vs. adult peripheral blood. Bone Marrow Transplant. 53, 1568-1577. https://doi.org/10.1038/s41409-018-0205-6 (2018).

17. Yu, X. et al. SENP3 maintains the stability and function of regulatory T cells via BACH2 deSUMOylation. Nat. Commun. 9, 3157. https://doi.org/10.1038/s41467-018-05676-6 (2018).

18. Kasakovski, D., Xu, L. \& Li, Y. T cell senescence and CAR-T cell exhaustion in hematological malignancies. J. Hematol. Oncol. 11, 91. https://doi.org/10.1186/s13045-018-0629-x (2018).

19. Kadereit, S. et al. Expansion of LTC-ICs and maintenance of p21 and BCL-2 expression in cord blood CD34(+)/CD38(-) early progenitors cultured over human MSCs as a feeder layer. Stem Cells 20, 573-582. https://doi.org/10.1634/stemcells.20-6-573 (2002).

20. Babenko, V. A. et al. Mirol enhances mitochondria transfer from multipotent mesenchymal stem cells (MMSC) to neural cells and improves the efficacy of cell recovery. Molecules https://doi.org/10.3390/molecules23030687 (2018).

21. Boukelmoune, N., Chiu, G. S., Kavelaars, A. \& Heijnen, C. J. Mitochondrial transfer from mesenchymal stem cells to neural stem cells protects against the neurotoxic effects of cisplatin. Acta Neuropathol. Commun. 6, 139. https://doi.org/10.1186/s40478-0180644-8 (2018).

22. Aggarwal, S. \& Pittenger, M. F. Human mesenchymal stem cells modulate allogeneic immune cell responses. Blood 105, 1815-1822. https://doi.org/10.1182/blood-2004-04-1559 (2005).

23. Zorn, E. et al. IL-2 regulates FOXP3 expression in human CD4+CD25+ regulatory T cells through a STAT-dependent mechanism and induces the expansion of these cells in vivo. Blood 108, 1571-1579. https://doi.org/10.1182/blood-2006-02-004747 (2006).

24. Beres, A., Komorowski, R., Mihara, M. \& Drobyski, W. R. Instability of Foxp3 expression limits the ability of induced regulatory T cells to mitigate graft versus host disease. Clin. Cancer Res. 17, 3969-3983. https://doi.org/10.1158/1078-0432.CCR-10-3347 (2011)

25. Rojewski, M. T., Weber, B. M. \& Schrezenmeier, H. Phenotypic characterization of mesenchymal stem cells from various tissues. Transfus Med. Hemother. 35, 168-184. https://doi.org/10.1159/000129013 (2008).

26. Bluestone, J. A. et al. Type 1 diabetes immunotherapy using polyclonal regulatory T cells. Sci. Transl. Med. 7, 315ra189. https:// doi.org/10.1126/scitranslmed.aad4134 (2015).

27. Lange, C., Scholl, M., Melms, A. \& Bischof, F. CD62L(high) Treg cells with superior immunosuppressive properties accumulate within the CNS during remissions of EAE. Brain Behav. Immun. 25, 120-126. https://doi.org/10.1016/j.bbi.2010.09.004 (2011).

28. van Leeuwen, M. A. et al. Changes in natural Foxp3(+)Treg but not mucosally-imprinted CD62L(neg)CD38(+)Foxp3(+)Treg in the circulation of celiac disease patients. PLoS One 8, e68432. https://doi.org/10.1371/journal.pone.0068432 (2013).

29. Lu, S. Y., Liu, K. Y., Liu, D. H., Xu, L. P. \& Huang, X. J. High frequencies of CD62L(+) naive regulatory T cells in allografts are associated with a low risk of acute graft-versus-host disease following unmanipulated allogeneic haematopoietic stem cell transplantation. Clin. Exp. Immunol. 165, 264-277. https://doi.org/10.1111/j.1365-2249.2011.04418.x (2011).

30. Tasso, R. et al. Mesenchymal stem cells induce functionally active T-regulatory lymphocytes in a paracrine fashion and ameliorate experimental autoimmune uveitis. Investig. Ophthalmol. Vis. Sci. 53, 786-793. https://doi.org/10.1167/iovs.11-8211 (2012).

31. Zheng, J. et al. ICOS regulates the generation and function of human CD4+ Treg in a CTLA-4 dependent manner. PLoS One 8, e82203. https://doi.org/10.1371/journal.pone.0082203 (2013).

32. MacDonald, K. N., Piret, J. M. \& Levings, M. K. Methods to manufacture regulatory T cells for cell therapy. Clin. Exp. Immunol. 197, 52-63. https://doi.org/10.1111/cei.13297 (2019).

33. Goodman, W. A. et al. IL-6 signaling in psoriasis prevents immune suppression by regulatory T cells. J. Immunol. 183, 3170-3176. https://doi.org/10.4049/jimmunol.0803721 (2009).

34. Xu, Y. et al. The E3 ligase Hrd1 stabilizes Tregs by antagonizing inflammatory cytokine-induced ER stress response. JCI Insight. https://doi.org/10.1172/jci.insight.121887 (2019).

35. Lee, S., Park, K., Kim, J., Min, H. \& Seong, R. H. Foxp3 expression in induced regulatory T cells is stabilized by C/EBP in inflammatory environments. EMBO Rep. https://doi.org/10.15252/embr.201845995 (2018).

36. Babenko, V. A. et al. Improving the post-stroke therapeutic potency of mesenchymal multipotent stromal cells by cocultivation with cortical neurons: The role of crosstalk between cells. Stem Cells Transl. Med. 4, 1011-1020. https://doi.org/10.5966/sctm. 2015-0010 (2015).

37. Leprat, P., Ratinaud, M. H. \& Julien, R. A new method for testing cell ageing using two mitochondria specific fluorescent probes. Mech. Ageing Dev. 52, 149-167 (1990).

38. Bukoreshtliev, N. V. et al. Selective block of tunneling nanotube (TNT) formation inhibits intercellular organelle transfer between PC12 cells. FEBS Lett. 583, 1481-1488. https://doi.org/10.1016/j.febslet.2009.03.065 (2009).

39. Marlein, C. R. et al. NADPH oxidase-2 derived superoxide drives mitochondrial transfer from bone marrow stromal cells to leukemic blasts. Blood 130, 1649-1660. https://doi.org/10.1182/blood-2017-03-772939 (2017).

40. Ahmad, T. et al. Miro1 regulates intercellular mitochondrial transport \& enhances mesenchymal stem cell rescue efficacy. EMBO J. 33, 994-1010. https://doi.org/10.1002/embj.201386030 (2014).

41. Kerkela, E. et al. Adenosinergic immunosuppression by human mesenchymal stromal cells requires co-operation with $\mathrm{T}$ cells. Stem Cells 34, 781-790. https://doi.org/10.1002/stem.2280 (2016).

42. Alam, M. S. et al. CD73 is expressed by human regulatory T helper cells and suppresses proinflammatory cytokine production and Helicobacter felis-induced gastritis in mice. J. Infect. Dis. 199, 494-504. https://doi.org/10.1086/596205 (2009).

43. Bowser, J. L. et al. Loss of CD73-mediated actin polymerization promotes endometrial tumor progression. J. Clin. Investig. 126, 220-238. https://doi.org/10.1172/JCI79380 (2016).

44. Antonioli, L., Pacher, P., Vizi, E. S. \& Hasko, G. CD39 and CD73 in immunity and inflammation. Trends Mol. Med. 19, 355-367. https://doi.org/10.1016/j.molmed.2013.03.005 (2013).

45. Allard, B., Longhi, M. S., Robson, S. C. \& Stagg, J. The ectonucleotidases CD39 and CD73: Novel checkpoint inhibitor targets. Immunol. Rev. 276, 121-144. https://doi.org/10.1111/imr.12528 (2017).

46. Liu, S. et al. Parkinson's disease-associated kinase PINK1 regulates Miro protein level and axonal transport of mitochondria. PLoS Genet. 8, e1002537. https://doi.org/10.1371/journal.pgen.1002537 (2012).

47. Kim, E. H. et al. Bach2 regulates homeostasis of Foxp3+ regulatory T cells and protects against fatal lung disease in mice. J. Immunol. 192, 985-995. https://doi.org/10.4049/jimmunol.1302378 (2014).

48. Watanabe-Matsui, M. et al. Heme regulates B-cell differentiation, antibody class switch, and heme oxygenase-1 expression in B cells as a ligand of Bach2. Blood 117, 5438-5448. https://doi.org/10.1182/blood-2010-07-296483 (2011).

49. Jang, K. J. et al. Mitochondrial function provides instructive signals for activation-induced B-cell fates. Nat. Commun. 6, 6750. https://doi.org/10.1038/ncomms7750 (2015).

50. Clayton, A., Al-Taei, S., Webber, J., Mason, M. D. \& Tabi, Z. Cancer exosomes express CD39 and CD73, which suppress T cells through adenosine production. J. Immunol. 187, 676-683. https://doi.org/10.4049/jimmunol.1003884 (2011).

51. Jeh, S. U. et al. Differential expression of the sirtuin family in renal cell carcinoma: Aspects of carcinogenesis and prognostic significance. Urol. Oncol. 35, 675e615-675e679. https://doi.org/10.1016/j.urolonc.2017.08.016 (2017).

52. Valencia, X. et al. TNF downmodulates the function of human CD4+CD25hi T-regulatory cells. Blood 108, 253-261. https://doi. org/10.1182/blood-2005-11-4567 (2006). 
53. Viglietta, V., Baecher-Allan, C., Weiner, H. L. \& Hafler, D. A. Loss of functional suppression by CD4+CD25+ regulatory T cells in patients with multiple sclerosis. J. Exp. Med. 199, 971-979. https://doi.org/10.1084/jem.20031579 (2004).

54. Kumar, M. et al. CD4+CD25+FoxP3+ T lymphocytes fail to suppress myelin basic protein-induced proliferation in patients with multiple sclerosis. J. Neuroimmunol. 180, 178-184. https://doi.org/10.1016/j.jneuroim.2006.08.003 (2006).

55. Hippen, K. L. et al. Generation and large-scale expansion of human inducible regulatory T cells that suppress graft-versus-host disease. Am. J. Transplant. 11, 1148-1157. https://doi.org/10.1111/j.1600-6143.2011.03558.x (2011).

56. Barber, D. L. et al. Restoring function in exhausted CD8 T cells during chronic viral infection. Nature 439, 682-687. https://doi. org/10.1038/nature04444 (2006)

57. Matsuzaki, J. et al. Tumor-infiltrating NY-ESO-1-specific CD8+ T cells are negatively regulated by LAG-3 and PD-1 in human ovarian cancer. Proc. Natl. Acad. Sci. U. S. A. 107, 7875-7880. https://doi.org/10.1073/pnas.1003345107 (2010).

58. Camisaschi, C. et al. LAG-3 expression defines a subset of CD4(+)CD25(high)Foxp3(+) regulatory T cells that are expanded at tumor sites. J. Immunol. 184, 6545-6551. https://doi.org/10.4049/jimmunol.0903879 (2010).

59. Holbrook, B. C. \& Alexander-Miller, M. A. Higher frequency and increased expression of molecules associated with suppression on $\mathrm{T}$ regulatory cells from newborn compared with adult nonhuman primates. J. Immunol. 205, 2128-2136. https://doi.org/10. 4049/jimmunol.2000461 (2020).

60. Falcone, M. \& Fousteri, G. Role of the PD-1/PD-L1 dyad in the maintenance of pancreatic immune tolerance for prevention of type 1 diabetes. Front. Endocrinol. 11, 569. https://doi.org/10.3389/fendo.2020.00569 (2020).

61. Chen, Y. et al. Glucocerebrosidase as a therapeutic target for Parkinson's disease. Expert Opin. Ther. Targets 24, 287-294. https:// doi.org/10.1080/14728222.2020.1733970 (2020).

62. Kato, R. et al. CD4(+)CD25(+)LAG3(+) T cells with a feature of Th17 cells associated with systemic lupus erythematosus disease activity. Front. Immunol. 10, 1619. https://doi.org/10.3389/fimmu.2019.01619 (2019).

63. Koenecke, C. et al. Alloantigen-specific de novo-induced Foxp3+ Treg revert in vivo and do not protect from experimental GVHD. Eur. J. Immunol. 39, 3091-3096. https://doi.org/10.1002/eji.200939432 (2009).

64. Someya, K. et al. Improvement of Foxp3 stability through CNS2 demethylation by TET enzyme induction and activation. Int. Immunol. 29, 365-375. https://doi.org/10.1093/intimm/dxx049 (2017).

65. Melief, S. M. et al. Multipotent stromal cells induce human regulatory T cells through a novel pathway involving skewing of monocytes toward anti-inflammatory macrophages. Stem Cells 31, 1980-1991. https://doi.org/10.1002/stem.1432 (2013).

66. Du, Y. M. et al. Mesenchymal stem cell exosomes promote immunosuppression of regulatory T cells in asthma. Exp. Cell Res. 363, 114-120. https://doi.org/10.1016/j.yexcr.2017.12.021 (2018).

67. Del Fattore, A. et al. Immunoregulatory effects of mesenchymal stem cell-derived extracellular vesicles on T lymphocytes. Cell Transplant. 24, 2615-2627. https://doi.org/10.3727/096368915X687543 (2015).

68. Court, A. C. et al. Mitochondrial transfer from MSCs to T cells induces Treg differentiation and restricts inflammatory response. EMBO Rep. https://doi.org/10.15252/embr.201948052 (2020).

69. Sidwell, T. et al. Attenuation of TCR-induced transcription by Bach2 controls regulatory T cell differentiation and homeostasis. Nat. Commun. 11, 252. https://doi.org/10.1038/s41467-019-14112-2 (2020).

70. Kunz, K., Piller, T. \& Muller, S. SUMO-specific proteases and isopeptidases of the SENP family at a glance. J. Cell Sci. https://doi. org/10.1242/jcs.211904 (2018).

71. Deaglio, S. et al. Adenosine generation catalyzed by CD39 and CD73 expressed on regulatory T cells mediates immune suppression. J. Exp. Med. 204, 1257-1265. https://doi.org/10.1084/jem.20062512 (2007).

72. Stagg, J. et al. CD73-deficient mice have increased antitumor immunity and are resistant to experimental metastasis. Cancer Res. 71, 2892-2900. https://doi.org/10.1158/0008-5472.CAN-10-4246 (2011).

73. Kobie, J. J. et al. T regulatory and primed uncommitted CD4 T cells express CD73, which suppresses effector CD4 T cells by converting 5'-adenosine monophosphate to adenosine. J. Immunol. 177, 6780-6786. https://doi.org/10.4049/jimmunol.177.10.6780 (2006).

74. Ehrentraut, H. et al. CD73+ regulatory T cells contribute to adenosine-mediated resolution of acute lung injury. FASEB J. 27, 2207-2219. https://doi.org/10.1096/fi.12-225201 (2013).

75. Quintero, O. A. et al. Human Myo19 is a novel myosin that associates with mitochondria. Curr. Biol. 19, 2008-2013. https://doi. org/10.1016/j.cub.2009.10.026 (2009).

76. Chang, K. T., Niescier, R. F. \& Min, K. T. Mitochondrial matrix Ca2+ as an intrinsic signal regulating mitochondrial motility in axons. Proc. Natl. Acad. Sci. U. S. A. 108, 15456-15461. https://doi.org/10.1073/pnas.1106862108 (2011).

77. Turrens, J. F. Mitochondrial formation of reactive oxygen species. J. Physiol. 552, 335-344. https://doi.org/10.1113/jphysiol.2003. 049478 (2003)

78. Byun, H. O., Kim, H. Y., Lim, J. J., Seo, Y. H. \& Yoon, G. Mitochondrial dysfunction by complex II inhibition delays overall cell cycle progression via reactive oxygen species production. J. Cell. Biochem. 104, 1747-1759. https://doi.org/10.1002/jcb.21741 (2008).

79. Schieke, S. M., McCoy, J. P. Jr. \& Finkel, T. Coordination of mitochondrial bioenergetics with G1 phase cell cycle progression. Cell Cycle 7, 1782-1787. https://doi.org/10.4161/cc.7.12.6067 (2008).

80. Weinberg, F. et al. Mitochondrial metabolism and ROS generation are essential for Kras-mediated tumorigenicity. Proc. Natl. Acad. Sci. U.S.A. 107, 8788-8793. https://doi.org/10.1073/pnas.1003428107 (2010).

81. Akkaya, B. et al. Increased mitochondrial biogenesis and reactive oxygen species production accompany prolonged CD4(+) T cell activation. J. Immunol. 201, 3294-3306. https://doi.org/10.4049/jimmunol.1800753 (2018).

82. Do, J. S. et al. Foxp3 expression in induced T regulatory cells derived from human umbilical cord blood vs. adult peripheral blood. Bone Marrow Transplant. https://doi.org/10.1038/s41409-018-0205-6 (2018).

83. Parmar, S. et al. Third-party umbilical cord blood-derived regulatory T cells prevent xenogenic graft-versus-host disease. Cytotherapy 16, 90-100. https://doi.org/10.1016/j.jcyt.2013.07.009 (2014).

84. Enomoto, M., Bunge, M. B. \& Tsoulfas, P. A multifunctional neurotrophin with reduced affinity to p75NTR enhances transplanted Schwann cell survival and axon growth after spinal cord injury. Exp. Neurol. 248, 170-182. https://doi.org/10.1016/j.expneurol. 2013.06.013 (2013).

85. Kitay, B. M., McCormack, R., Wang, Y., Tsoulfas, P. \& Zhai, R. G. Mislocalization of neuronal mitochondria reveals regulation of Wallerian degeneration and NMNAT/WLD(S)-mediated axon protection independent of axonal mitochondria. Hum. Mol. Genet. 22, 1601-1614. https://doi.org/10.1093/hmg/ddt009 (2013).

86. Park, S. W., Pyo, C. W. \& Choi, S. Y. High-efficiency lentiviral transduction of primary human CD34(+) hematopoietic cells with low-dose viral inocula. Biotechnol. Lett. 37, 281-288. https://doi.org/10.1007/s10529-014-1678-z (2015).

87. Sonntag, K. et al. Chronic graft-versus-host-disease in CD34(+)-humanized NSG mice is associated with human susceptibility HLA haplotypes for autoimmune disease. J. Autoimmun. 62, 55-66. https://doi.org/10.1016/j.jaut.2015.06.006 (2015).

88. Ito, R. et al. Highly sensitive model for xenogenic GVHD using severe immunodeficient NOG mice. Transplantation 87, $1654-1658$. https://doi.org/10.1097/TP.0b013e3181a5cb07 (2009).

89. Erben, U. et al. A guide to histomorphological evaluation of intestinal inflammation in mouse models. Int. J. Clin. Exp. Pathol. 7, 4557-4576 (2014). 


\section{Acknowledgements}

We thank Abigail Hotchner and Meryl Kovacs for assistance with cell culture and Dr. Helena Reijonen for editorial assistant and valuable suggestions. The authors gratefully acknowledge the Char and Chuck Fowler Foundation, Cleveland, $\mathrm{OH}$ and the Abraham J. \& Phyllis Katz Foundation, Atlanta, GA for supporting this work.

\section{Author contributions}

J.D., D.Z., J.K., F.Z., D.A., M.J.L. conducted experiments, literature search, developed study design, and analyzed data. J.D., D.Z., J.K., and M.J.L. wrote the manuscript. A.H., W.H., M.F. contributed to data review and interpretation, as well as review and approval of the manuscript.

\section{Competing interests}

The authors declare no competing interests.

\section{Additional information}

Supplementary Information The online version contains supplementary material available at https://doi.org/ 10.1038/s41598-021-90115-8.

Correspondence and requests for materials should be addressed to J.D.

Reprints and permissions information is available at www.nature.com/reprints.

Publisher's note Springer Nature remains neutral with regard to jurisdictional claims in published maps and institutional affiliations.

Open Access This article is licensed under a Creative Commons Attribution 4.0 International License, which permits use, sharing, adaptation, distribution and reproduction in any medium or format, as long as you give appropriate credit to the original author(s) and the source, provide a link to the Creative Commons licence, and indicate if changes were made. The images or other third party material in this article are included in the article's Creative Commons licence, unless indicated otherwise in a credit line to the material. If material is not included in the article's Creative Commons licence and your intended use is not permitted by statutory regulation or exceeds the permitted use, you will need to obtain permission directly from the copyright holder. To view a copy of this licence, visit http://creativecommons.org/licenses/by/4.0/.

(C) The Author(s) 2021 